\title{
C. elegans establishes germline versus soma by balancing inherited histone methylation
}

Brandon S. Carpenter1, Teresa W. Lee1, Caroline F. Plott2, Jovan S. Brockett3, Dexter A. Myrick1, David J. Katz1*

1Department of Cell Biology, Emory University School of Medicine, Atlanta GA 30322, USA. 2Johns Hopkins University School of Medicine, Baltimore MD 21205, USA.

3Department of Biology, Oglethorpe University, Atlanta GA 30319, USA.

*corresponding author:

David J. Katz

Associate Professor

Department of Cell Biology

Room 443, Whitehead Biomedical Research Building

Emory University School of Medicine

Atlanta, GA 30322, USA

Phone: (404) 727-3403

djkatz@emory.edu 


\section{ABSTRACT}

2 Embryos undergo extensive reprogramming at fertilization to prevent the inappropriate

3 inheritance of histone methylation. In C. elegans, this reprogramming is mediated by the

4 H3K4me2 demethylase, SPR-5, and the H3K9 methyltransferase, MET-2. In contrast to this

5 reprogramming, the H3K36 methyltransferase, MES-4, maintains H3K36me2/3 at germline

6 genes between generations to help re-establish the germline. To determine whether the

7 MES-4 germline inheritance system antagonizes spr-5; met-2 reprogramming, we examined

8 the interaction between these two systems. We find that the developmental delay of $s p r-5$;

9 met-2 mutant progeny is associated with ectopic H3K36me2/3 and the ectopic expression

10 of MES-4 targeted germline genes in somatic tissues. Furthermore, the developmental

11 delay is dependent upon MES-4 and the H3K4 methyltransferase, SET-2. We propose that

12 the MES-4 inheritance system prevents critical germline genes from being repressed by

13 maternal spr-5; met-2 reprogramming. Thus, the balance of inherited histone modifications

14 is necessary to distinguish germline versus soma and prevent developmental delay.

INTRODUCTION

In multicellular organisms, developmental cell fate decisions are established by

18 tightly controlled spatial and temporal gene expression. This pattern of gene expression

19 can be regulated by heritable modifications on the $\mathrm{N}$-terminal tails of histone proteins

20 (Jambhekar et al., 2019). For example, methylation of either lysine 4 or 36 on histone 3,

21 (H3K4me and H3K36me) is generally associated with active transcription, whereas

22 methylation of lysine 9 on the same histone (H3K9me) is commonly associated with

23 transcriptional repression. These modifications are dynamically regulated by the specific 
24 and tightly controlled activity of histone modifying enzymes. For instance, mono- and di-

25 methylation of lysine 4 on histone $\mathrm{H} 3(\mathrm{H} 3 \mathrm{~K} 4 \mathrm{me} 1 / 2)$ are removed by the demethylase

26 LSD1/KDM1A (Y. Shi et al., 2004; Y.-J. Shi et al., 2005). In the nematode C. elegans,

27 populations of mutants lacking the LSD1 ortholog, SPR-5, become increasingly sterile over

$28 \sim 30$ generations (Katz et al., 2009). Failure to erase H3K4me2 at fertilization between

29 generations in spr-5 mutants correlates with an accumulation of H3K4me2 and

30 spermatogenesis gene expression across 30 generations, which leads to increasing sterility

31 (Katz et al., 2009). These data demonstrate that H3K4me2 can function as an epigenetic

32 transcriptional memory. More recently, we demonstrated that SPR-5 synergizes with the

33 H3K9me2 methyltransferase, MET-2, to regulate maternal epigenetic reprogramming (Kerr

34 et al., 2014). Progeny of mutants lacking both SPR-5 and MET-2 suffer from developmental

35 delay and become completely sterile in a single generation (Kerr et al., 2014). These

36 phenotypes are associated with synergistic increases in both H3K4me2 and candidate

37 germline gene expression in somatic tissues (Kerr et al., 2014). Together this work

38 supports a model in which SPR-5 and MET-2 are maternally deposited into the oocyte,

39 where they reprogram histone methylation to prevent inherited defects.

40 Following fertilization, the C. elegans embryo separates germline versus somatic

41 lineages progressively through a series of asymmetric divisions (Strome, 2005). To

42 accomplish this, transcription factors coordinate with multiple histone modifications. For

43 example, maternal deposition of PIE-1, a germline specific protein that asymmetrically

44 segregates into germline blastomeres (P lineage cells), maintains the fate of germ cells by

45 inhibiting POL-II elongation and preventing the ectopic expression of somatic genes

46 (Batchelder et al., 1999; Mello et al., 1992; Seydoux et al., 1996). In the absence of 
47 transcription in the germline, the maternally provided H3K36me2/3 methyltransferase,

48 MES-4, binds to a subset of germline genes that were previously expressed in the parental

49 germline (Furuhashi et al., 2010; Rechtsteiner et al., 2010). These germline genes are

50 recognized by MES-4 via H3K36me2/3 that was added in the parental germline by the

51 transcription-coupled H3K36me2/3 methyltransferase, MET-1 (Kreher et al., 2018). MES-4

52 maintains H3K36me2/3 at these genes in the early embryo in a transcriptionally

53 independent manner. Without maternally deposited MES-4, the germline cannot properly

54 proliferate and animals are sterile (Capowski et al., 1991; Garvin et al., 1998). For the

55 remainder of this study, we will refer to these MES-4 targeted genes as MES-4 germline

56 genes, and the process through which the MES-4 germline inheritance system maintains

57 these genes for re-activation in the offspring as bookmarking.

MES-4 bookmarking is antagonized in somatic tissues by transcriptional repressors

59 and chromatin remodelers. For example, loss of the transcriptional repressors LIN-15B and

LIN-35 at high temperatures leads to larval arrest (Petrella et al., 2011). This larval arrest

61 can be suppressed by removing the MES-4 germline inheritance system (Petrella et al.,

62 2011). Removing the MES-4 inheritance system also suppresses the somatic expression of

63 germline genes in lin-35 mutants (Wang et al., 2005). Similar to LIN-15B and LIN-35, loss of

64 the chromatin remodelers MEP-1 and LET-418 causes somatic expression of germline

65 genes and larval arrest (Unhavaithaya et al., 2002). This somatic expression of germline

66 genes and larval arrest is also dependent upon the MES-4 germline inheritance system

67 (Unhavaithaya et al., 2002). Together, these findings demonstrate that transcriptional

68 repressors and the chromatin remodelers function in somatic tissues to antagonize H3K36

69 bookmarking by MES-4. 
Recently, the repressive histone modification H3K9me2 has also been implicated in

71 the somatic repression of germline genes (Rechtsteiner et al., 2019). Some germline genes

72 have H3K9me2 enrichment at their promoters in somatic tissues (Rechtsteiner et al.,

73 2019). Loss of LIN-15B reduces this enrichment of H3K9me2 leading to the ectopic

74 accumulation of H3K36me3 at gene bodies in somatic tissues (Rechtsteiner et al., 2019).

75 This raises the possibility that LIN-15B may antagonize the MES-4 germline inheritance in

76 part through the repressive histone modification $\mathrm{H} 3 \mathrm{~K} 9 \mathrm{me} 2$.

77 Despite the extensive knowledge of the systems that somatically antagonize the

78 MES-4 germline inheritance system, it remains unclear why germline genes are

79 bookmarked by H3K36 in the embryo. To address this major gap, we examined somatic

80 development in progeny deficient in SPR-5 and MET-2 maternal reprogramming. Our

81 previous work suggests that maternal spr-5; met-2 reprogramming prevents the

82 transgenerational inheritance of H3K4me2 by erasing this mark and coupling it to the

83 acquisition of H3K9me2 between generations (Kerr et al., 2014). Here we show that

84 H3K36me3 ectopically accumulates at MES-4 germline genes in the somatic tissues of spr-

85 5; met-2 double mutant progeny (hereafter referred to as spr-5; met-2 progeny), and this

86 accumulation correlates with the ectopic expression of these genes. In addition, we find

87 that both the developmental delay and the ectopic expression of germline genes is rescued

88 by knocking down MES-4 activity. These data provide comprehensive evidence that the

89 ectopic expression of MES-4 targeted germline genes in somatic tissues causes

90 developmental delay. In addition, we demonstrate that the severe developmental delay of

91 spr-5; met-2 progeny is rescued by the loss of the H3K4 methyltransferase SET-2. This

92 finding suggests that the ectopic maintenance of the MES-4 germline inheritance system in 
93 spr-5; met-2 progeny is driven by the inheritance of H3K4 methylation. Finally, by

94 demonstrating that loss of maternal spr-5; met-2 reprogramming unbalances the MES-4

95 germline inheritance system, our data suggest that H3K36 methylation bookmarking

96 functions to antagonize spr-5; met-2 maternal reprogramming. Thus, we propose that $C$.

97 elegans balances three different histone modifications to distinguish between the

98 competing fates of soma and germline.

\section{RESULTS}

\section{Loss of spr-5 and met-2 causes a severe developmental delay}

105 hermaphrodites and monitored their development from hatching to adults. By 72 hours, all N2 progeny (469/469), most of the spr-5 progeny (363/385), and many of met-2

107 progeny (386/450) were fertile adults (Figure 1A, B, C, and E, Figure 1-figure supplement

108 1A-C). In contrast, spr-5; met-2 progeny displayed a severe developmental delay, with none 109 of the progeny (0/463) reaching adulthood by 72 hours (Figure 1D, E; and Figure 1-figure 110 supplement 1D). The majority of spr-5; met-2 progeny (371/463) resembled L2 larvae at

11172 hours, while a smaller percentage of the population developed to later larval stages

112 (42/463) (supplementary file 1). By seven days post synchronized lay, a small number of 113 spr-5; met-2 progeny (35/876) developed into adults and the majority (31/35) of these

114 adults displayed protruding vulva (Pvl) (Figure 1-figure supplement 1E-G). All 35 of the 115 spr-5; met-2 mutant progeny that developed to adulthood were sterile. 


\section{MES-4 germline genes are ectopically expressed in spr-5; met-2 mutant soma}

Previously we showed that H3K4me2 is synergistically increased in spr-5; met-2

123 To do this, we performed RNA-seq on spr-5; met-2 L1 progeny compared to N2 (wild-type)

124 L1 progeny. We chose to perform this analysis on L1 larvae because this stage immediately 125 precedes the L2 larval delay that we observe in spr-5; met-2 progeny (see Figure 1A, D). In 126 addition, L1 larvae are composed of 550 somatic cells and two germ cells. Therefore, L1

127 larvae are primarily composed of somatic tissue. As a control, we also performed RNA-seq 128 on L1 progeny from spr-5 and met-2 single mutants that were isolated from early generation animals, within the first five generations. These generations are well before the onset of sterility that we previously reported (Katz et al., 2009; Kerr et al., 2014).

134 spr-5 (159/413) and met-2 single mutants (113/343) compared to N2 (Figure 2-

135 supplement figure 1A-B, Figure 2-supplement figure 2A, B, D, E, and supplementary file 2).

136 Gene Set Enrichment Analysis (GSEA) did not identify any categories of genes

137 misexpressed in the spr-5 or met-2 single mutants. However, GSEA revealed that genes

138 differentially expressed in spr-5; met-2 progeny were significantly enriched (based on 
139 Combined Score, (Chen et al., 2013)) for biological processes and cellular components

140 characteristic of the germline; including meiosis, P-granules and negative regulation of the

141 cell cycle (Figure 2-supplement figure 1C-D). Many of the genes involved in these processes

142 are expressed in the germline of the parental generation, bound by the H3K36

143 methyltransferase MES-4 in the early embryo, and marked by H3K36me2/3, independent

144 of POL-II (Rechtsteiner et al., 2010). As a result, we were interested in the potential overlap

145 with MES-4 targeted genes (referred to as MES-4 germline genes).

147 (Rechtsteiner et al., 2010). We reasoned that the absence of SPR-5 and MET-2

148 reprogramming may cause germline genes to be aberrantly targeted by MES-4 in the soma,

149 leading to ectopic expression. To investigate this possibility, we examined the overlap

150 between differentially expressed genes in spr-5; met-2 L1 progeny and MES-4 germline

151 genes. Out of 196 MES-4 germline genes, 34 overlapped with genes up-regulated in spr-5;

152 met-2 progeny compared to N2 (Figure 2A, hypergeometric test, p-value $<6.44 \mathrm{e}-20$ ), while

153 zero overlapped with genes down-regulated in spr-5; met-2 progeny compared to N2

154 (Figure 2B). In addition, when we compared the log2 fold change (FC) in expression of all of

155 the MES-4 germline genes in spr-5, met-2, and spr-5; met-2 mutant progeny compared to

156 N2, we observed that 108 of the MES-4 germline genes were synergistically increased in

157 spr-5; met-2 progeny compared to single mutant progeny (Figure 2C and supplementary

158 file 3).

159 During this initial RNA-seq analysis we had to genotype every spr-5; met-2 L1

160 because the balancer chromosome that was available did not completely balance spr-5. As a

161 result, the RNA-seq was performed using a low-input sequencing technique (see methods). 
162 However, during the course of the experiments, a new balancer became available that

163 completely balances spr-5. This enabled us to repeat the spr-5; met-2 RNA-seq experiments

164 using standard amounts of RNA. In the repeat spr-5; met-2 RNA-seq experiment, we

165 identified significantly more differentially expressed genes compared to N2 (4223 vs. 778

166 in the initial low-input analysis). However, despite the larger number of differentially

167 expressed genes, MES-4 germline genes remained similarly enriched. Out of 196 MES-4

168 germline genes, 112 overlapped with genes up-regulated in spr-5; met-2 progeny compared

169 to N2 (Figure 2-supplemental figure 3A, hypergeometric test, P-value $<1.20 \mathrm{e}-54$, and

170 supplementary file 2), while only two overlapped with genes down-regulated in spr-5; met-

1712 progeny compared to N2 (Figure 2-supplemental figure 3B). We also compared the

$172 \log$ FC in expression of all of the MES-4 germline genes in spr-5; met-2 mutant progeny

173 compared to N2. This analysis revealed that the MES-4 germline genes were similarly

174 overexpressed in spr-5; met-2 progeny compared to N2 (Figure 2D and supplementary file

175 3). Interestingly, while MES-4 germline genes were enriched in both spr-5; met-2 RNA-seq

176 experiments, there were some differences in the specific MES-4 germline genes that were

177 overexpressed, and the extent to which they were overexpressed (Figure 2-supplemental

178 figure 3C and supplementary file 3).

179

180 smFISH confirmation of MES-4 germline gene expression in spr-5; met-2 mutant

181 soma

To confirm that MES-4 germline genes are somatically expressed in spr-5; met-2 L1

183 progeny, we performed single molecule fluorescent in situ hybridization (smFISH) on two

184 MES-4 germline targets, $h t p-1$ and $c p b-1$ (Figure 3). Both of these genes were amongst the 
genes that were ectopically expressed in spr-5; met-2 L1 progeny compared to N2 L1

restricted to the two primordial germ cells, $\mathrm{Z} 2$ and $\mathrm{Z3}$, which go on to form the entire adult

contrast, in spr-5; met-2 progeny htp-1 was ectopically expressed throughout the soma

(Figure 3D-F). This expression pattern is similar to what we observed with the

ubiquitously expressed subunit of RNA polymerase II, ama-1 (Figure 3-supplement figure

\section{MES-4 germline genes maintain ectopic H3K36me3 in spr-5; met-2 mutants}

To test whether MES-4 germline genes that are ectopically expressed in the soma of

$203 Y 18 D 10 A .11$, and $f b x a-101$, that are ectopically expressed in our RNA-seq analysis, showed

204 increased levels of H3K36me3 in spr-5; met-2 progeny (Figure 4J-M; Figure 4-supplement 205 figure 1J-M) compared to N2 progeny (Figure 4C-F; Figure 4-supplement figure 1C-F). As a 
nerve chord in L1 progeny (Figure 4G, N; Figure 4-supplement figure 1G, N), ama-1, a

supplement figure $1 \mathrm{H}, 0$ ), and act-1, a ubiquitously expressed actin related protein (Figure

211 4I, P; Figure 4-supplement figure 1I, P). Each of these control genes displayed similar

212 H3K36me3 enrichment in both spr-5; met-2 and N2 L1 progeny (compare Figure 4N-P and

213 Figure 4-supplement figure 1N-P to Figure 4G-I and Figure 4-supplement figure 1G-I,

214 respectively).

MES-4 germline genes display H3K9me2 at their promoter peaks

222 would expect MES-4 germline genes that are ectopically expressed in the somatic tissues of

223 spr-5; met-2 progeny to normally continue to be targeted by H3K9 methylation in these

224 tissues. To examine this possibility, we re-analyzed L1 stage H3K9me2 Chip-seq data from

225 Rechsteiner et al. 2019 (Rechtsteiner et al., 2019). This re-analysis showed that many of the

226 MES-4 germline genes were enriched for H3K9me2 at their promoters (Figure 4-

227 supplement figure 2A), including the majority of MES-4 germline genes that were

228 ectopically expressed in the soma of spr-5; met-2 progeny (Figure 4-supplement figure 2B).

229 For example, the MES-4 germline genes $c p b-1, T 05 B 9.1, Y 18 D 10 A .11$, and $f b x a-101$, that 
231 of spr-5; met-2 progeny, had H3K9me2 peaks at their promoters (Figure 4-supplement

232 figure 2C-F). In contrast, our control genes ceh-13, ama-1, and act-1, that were not

233 misexpressed, were also not enriched for H3K9me2 at their promoters (Figure 4-

234 supplement figure 2G-I).

235

236

Knocking down MES-4 rescues ectopic expression of germline genes in spr-5; met-2

mutant soma

To test whether the ectopic expression of MES-4 germline genes in spr-5; met-2

239 progeny is dependent on the ectopic H3K36me3, we examined whether the expression of

240 these genes was dependent upon MES-4. We performed quantitative real-time PCR (qRT-

241 PCR) on L1 progeny from spr-5; met-2 hermaphrodites fed control (L4440) RNAi versus

242 mes-4 RNAi (Figure 5). For this analysis, we selected candidate MES-4 germline genes that

243 were ectopically expressed and displayed an ectopic H3K36me3 peak in spr-5; met-2 L1

244 progeny compared to N2 L1 progeny. Consistent with our RNA-seq analysis, all nine of the

245 candidate MES-4 germline genes that we examined were ectopically expressed $>2$ fold in

246 spr-5; met-2 L1 progeny compared to control (Figure 5). Strikingly, the ectopic expression

247 of the nine MES-4 candidate germline genes was dependent upon MES-4. Nine out of nine

248 of these genes were significantly decreased in L1 progeny from spr-5; met-2

249 hermaphrodites treated with mes-4 RNAi (Figure 5; unpaired t-test, p-value < 0.001), and

250 all but one (T05B9.1) were reduced to levels that were similar to N2 L1 progeny.

252 Knocking down MES-4 rescues developmental delay in spr-5; met-2 progeny 
To test whether the developmental delay phenotype that we observe in spr-5; met-2

254 progeny is also dependent on the ectopic expression of MES-4 germline genes, we fed spr-5;

255 met-2 hermaphrodites mes-4 RNAi and monitored their progeny for 72 hours after a

256 synchronized lay. If the developmental delay is dependent upon the ectopic expression of

257 MES-4 germline genes, it should be suppressed when this ectopic expression is eliminated

258 via mes-4 RNAi. By 72 hours, all of the N2 control progeny from hermaphrodites fed either

259 L4440 control (1089/1089) or mes-4 (1102/1102) RNAi were adults (Figure 6A, B, I). Also,

260 consistent with our previous observations, all but one of the spr-5; met-2 mutant progeny

261 (729/730) from hermaphrodites fed control RNAi remained in the L2-L3 larval stages

262 (Figure 6E, I). In contrast, most of spr-5; met-2 progeny (569/618) from hermaphrodites

263 fed mes-4 RNAi developed to adults (Figure 6F, I, unpaired t-test, $\mathrm{p}<0.0001$ ). Though as

264 expected, these animals remained sterile due to the mes-4 RNAi preventing any germline

265 formation.

266

MET-1 mediates transcription-coupled H3K36me3 in the adult germline and in later

267 stage transcriptionally active embryos (Furuhashi et al., 2010; Kreher et al., 2018;

268 Rechtsteiner et al., 2010). If the ectopic somatic expression of MES-4 germline genes is

269 caused by the transcription independent maintenance of H3K36me3 via MES-4, we would

270 expect the developmental delay to be independent of MET-1. To determine whether the

271 developmental delay phenotype in spr-5; met-2 progeny is dependent on MET-1, we

272 monitored the development of progeny from spr-5; met-2 hermaphrodites fed met-1 RNAi

273 for 72 hours after a synchronized lay. In contrast to the suppression that we observed upon

274 mes-4 RNAi, met-1 RNAi had no effect. By 72 hours, none of the spr-5; met-2 mutant

275 progeny (0/573) from hermaphrodites fed met-1 RNAi developed to adults, and these 
276 animals were indistinguishable from spr-5; met-2 progeny fed control RNAi (Figure 6G, I).

277 Additionally, met-1 RNAi itself had no effect on developmental delay, as nearly all of the N2

278 progeny (715/721) from hermaphrodites fed met-1 RNAi developed to adults (Figure 6C,

279 I).

280

281

Knocking down SET-2 rescues spr-5; met-2 developmental delay

If the developmental delay of spr-5; met-2 mutants is caused by ectopic inheritance

283

of H3K4me2 driving the expression MES-4 germline genes in somatic tissues, we would

284

expect the developmental delay of spr-5; met-2 progeny to be dependent upon the H3K4

285

methyltransferase, SET-2. To test this, we monitored the development of progeny of spr-5;

met-2 hermaphrodites fed set-2 RNAi for 72 hours after a synchronized lay. Identical to N2

287 progeny from hermaphrodites fed control RNAi, set-2 RNAi had no effect on the

288 development of wild-type animals, as all of the N2 progeny from hermaphrodites fed set-2

289 RNAi developed to adults by 72 hours (1114/1114) (Figure 6D, I). However, in contrast to

290 spr-5; met-2 progeny fed control RNAi that were developmentally delayed, most of the

291 progeny from spr-5; met-2 hermaphrodites fed set-2 RNAi developed to adults (347/384)

292 (Figure 6H, I, unpaired t-test, $\mathrm{p}<0.0001$ ).

293

spr-5; met-2 progeny acquire transgene silencing in somatic tissues

The somatic expression of MES-4 germline genes involved in germline transgene

296 silencing (Figure S2C-D) raises the possibility that the somatic tissues in spr-5; met-2

297 progeny may acquire the ability to silence transgenes, a function normally restricted to

298 germline cells. To test this, we examined the somatic expression of an extrachromosomal 
299

300

301

302

303

304

305

306

307

308

309

310

311

312

313

314

315

316

317

318

319

320

\section{DISCUSSION}

multicopy let-858 transgene that is normally silenced in the germline by both transcriptional and posttranscriptional germline silencing mechanisms (Kelly and Fire, 1998). This analysis was performed in spr-5; met-2 mutant L2 larvae that were undergoing developmental delay. In N2, most of the L2 progeny (117/132) expressed ubiquitous high levels of LET-858::GFP throughout the entire soma (Figure 7A, B, and K), while the remaining progeny (15/132) expressed what we describe as a "faint" level of expression (Figure 7C, D, and K). In contrast, almost none of the spr-5; met-2 L2 progeny (2/87) displayed high level of transgene expression that is comparable to the high level seen in most N2 progeny (Figure 7E, F, and K). Instead, the majority of spr-5; met-2 progeny (64/87) had faint LET-858::GFP expression that is comparable to the faint expression observed in N2 progeny (Figure 7G, H, and K). The remaining 21 spr-5; met-2 progeny had no LET-858::GFP expression. Because less than 50\% of progeny inherited the let-858 transgene, we normalized the percentage of progeny scored as "off" for LET-858::GFP to the presence of the let-858 transgene based on genotyping for $g f p$ (see methods). For N2, all of the $60 \mathrm{~L} 2$ progeny that were scored as "off" failed to inherit the let-858 transgene, indicating that the transgene is never silenced in N2 progeny (data not shown). However, for spr-5; met-2 L2 progeny, eight out of 50 progeny that were scored as "off" inherited the let-858 transgene indicating that the let-858 transgene can be completely silenced in some spr-5; met-2 progeny. After normalization for the transgene inheritance, we observed that 21/87 of spr-5; met-2 progeny displayed no visible expression of the LET-858::GFP transgene (Figure 7I, J, and K). 
spr-5; met-2 maternal reprogramming prevents developmental delay by restricting ectopic MES-4 bookmarking

SPR-5 and MET-2 act maternally to reprogram histone methylation and prevent the transcriptional state of the parent from being inappropriately transmitted to the offspring (Kerr et al., 2014). In this study, we find that the loss of SPR-5 and MET-2 maternal reprogramming led to a severe developmental delay that was associated with the ectopic expression of MES-4 germline genes in somatic tissues. This finding raises the possibility that SPR-5 and MET-2 reprogramming blocks the ectopic expression of MES-4 germline genes by preventing the accumulation of MES-4 dependent H3K36me3 at a subset of 331 germline genes in somatic tissues. Consistent with this possibility, most of the MES-4

332 germline genes were increased in spr-5; met-2 mutants compared to N2 in L1 larvae. Using 333 smFISH, we also confirmed the somatic expression of two ectopically expressed MES-4

334 germline genes, $h t p-1$ and $c p b-1$. While $h t p-1$ mRNA was ectopically detected in many somatic tissues, the ectopic expression of $c p b-1$ mRNA was more restricted, suggesting that 336 the extent of ectopic expression is dependent upon the locus. In the absence of SPR-5 and MET-2 reprogramming, MES-4 germline genes

338 accumulated ectopic H3K36me3 in the soma. This suggests that without SPR-5 and MET-2 339 reprogramming, MES-4 ectopically maintains H3K36me3 at these genes in somatic tissues.

340 Of note, we observe a low level of H3K36me3 at germline genes in the somatic tissues of N2

341 progeny. It is unclear why there is a low level of H3K36me3 normally in somatic tissues in

342 N2 animals. Nevertheless, the absence of transcription associated with this low level of 343 H3K36me3 indicates that an increased level of H3K36me3 is necessary to cause ectopic 344 transcription. 
If the ectopic maintenance of H3K36me3 in the soma of spr-5; met-2 mutant progeny expression and developmental delay. Indeed, we find that the removal of MES-4 rescued and the developmental delay. Importantly, removing the transcriptionally coupled the ectopic expression of MES-4 germline genes.

How does an ectopic transcriptional program interfere with developmental timing?

How does the ectopic expression of germline genes interfere with somatic tissues to

germline genes causes the soma to take on germline character. To begin to address this, we

asked whether spr-5; met-2 double mutants can silence an extrachromosomal array in

somatic tissues. The silencing of extrachromosomal arrays is normally restricted to the

ability to silence an extrachromosomal multicopy array in somatic cells. Consistent with

365 GFP transgene (Wang et al., 2005). Intriguingly, in the spr-5; met-2 mutant RNA-seq we 
pathways have previously been implicated in gene silencing (Buckley et al., 2012; Sijen et

(Wang et al., 2005), it is possible that the somatic silencing of the transgene in spr-5; met-2

372 heritable silencing via small RNAs requires MET-2. This provides a further indication that

373 the chromatin and small RNA pathways may be functioning together (Lev et al., 2017).

Normally in L1 larvae, the primordial germ cells, Z2 and Z3, are arrested at the

377 and the cell cycle, as well as G2/M checkpoint genes. Thus, it is also possible that ectopic

378 expression of germline genes normally expressed only in Z2 and Z3 contributes to the

379 developmental delay through the ectopic activation of germline cell cycle control.

380 Regardless, the silencing of the extrachromosomal multicopy array suggests that the

381 somatic tissues in spr-5; met-2 progeny make functional proteins that can perform some

382 germline functions. We propose that either ectopic germline transcription, or an ectopic

383 germline function resulting from this ectopic transcription, interferes with the ability of

384 somatic cells to properly enact their transcriptional program. This background noise delays

385 the proper adoption of cell fate, leading to an overall delay in the development of the tissue.

387 A model for how the inheritance of histone methylation is balanced to specify

388 germline versus soma

By linking maternal spr-5; met-2 reprogramming to the MES-4 germline inheritance

390 system, our data provide a rationale for the existence of MES-4 bookmarking, through the 
391 following model. In the germline, transcriptional elongation is blocked by PIE-1, which

392 segregates to germline blastomeres during embryogenesis (Batchelder et al., 1999; Mello et

393 al., 1992; Seydoux et al., 1996) (Figure 8A, B). In the soma of the early embryo, there is also

394 very little transcription, because the bulk of zygotic transcription does not begin until

395 approximately the 60-cell stage. This stage is just prior to when the primordial germ cells,

396 Z2 and Z3, are specified (Sulston et al., 1983). Thus, in C. elegans, germline versus soma is

397 largely specified without transcription.

During normal maternal reprogramming, SPR-5 and MET-2 are deposited into the oocyte. At fertilization they facilitate the reprogramming of previously expressed genes from an active chromatin state to a repressed chromatin state by removing H3K4me2 and

401 adding H3K9me2 (Kerr et al., 2014) (Figure 8A). This reprogramming is necessary to 402 prevent the transcriptional memory of the previous generation from being inappropriately 403 propagated to the progeny. Genes epigenetically reprogrammed by SPR-5 and MET-2 404 include ubiquitously expressed genes and germline expressed genes, a subset of which are

405 MES-4 germline genes. The MES-4 germline genes are subsequently targeted by the 406 transcription independent H3K36 methyltransferase, MES-4, to maintain H3K36me3 in the 407 germ lineage during embryogenesis (Figure 8A, B). We propose that H3K36 methylation 408 bookmarking antagonizes the repression caused by the erasure of H3K4me2 and the 409 addition of H3K9me2. Without the transcription independent maintenance of inherited 410 H3K36 methylation from the mother to antagonize this repression, the germline fails to 411 proliferate and animals are sterile (Capowski et al., 1991; Garvin et al., 1998). The failure to 412 proliferate and sterility caused by loss of MES-4 is presumably because germline genes that 413 are targeted by MES-4 fail to reactivate, though it has yet to be demonstrated. Thus, our 
414 data suggest that the MES-4 bookmarking system is necessary for critical germline genes to

415 bypass the global epigenetic reprogramming that occurs at fertilization to prevent

416 transgenerational inheritance. We refer to this initial phase of filtering inherited histone

417 methylation at fertilization as the establishment phase.

418 Following this establishment phase, a maintenance phase is required to propagate

419 this initial pattern of histone methylation throughout embryogenesis. The MES-4

420 bookmarking system is localized primarily to the primordial germ cells in later embryonic

421 development (Furuhashi et al., 2010; Rechtsteiner et al., 2010). This concentration of MES-

4224 to the germline helps to maintain MES-4 bookmarking for germline specification later in

423 embryonic development. However, MES-4 is also present in somatic tissues (Furuhashi et

424 al., 2010; Rechtsteiner et al., 2010). This makes the system vulnerable to the ectopic

425 maintenance of MES-4 bookmarking in the soma. For this reason, multiple systems, such as

426 LIN-15B and LIN-35, as well as MEP-1 and LET-418, function in somatic tissues to restrict

427 H3K36 bookmarking by MES-4 to the germline. Thus, in the maintenance phase, the

428 balance of MES-4 and the systems that somatically antagonize MES-4, maintain the histone

429 methylation pattern that is initiated during the establishment phase. Taken together, we

430 propose that SPR-5, MET-2 and MES-4 carefully balance the inheritance of three different

431 histone modifications, H3K4, H3K9, and H3K36 methylation, to ensure the proper

432 specification of germline versus soma in the absence of transcription.

433 The model that we have proposed makes the following two predictions. First, MES-4

434 germline genes should normally be targeted for continued silencing by H3K9me2 in

435 somatic tissues. It has recently been shown that a subset of germline specific genes contain

436 H3K9me2 at their promoters in somatic tissues (Rechtsteiner et al., 2019). We re-examined 
437 the H3K9me2 ChIP-seq dataset from this work and found that the MES-4 germline genes

438 that are ectopically expressed in the somatic tissues of spr-5; met-2 progeny also displayed

439 unique H3K9me2 promoter peaks. This confirms that MES-4 germline genes are normally

440 repressed by $\mathrm{H} 3 \mathrm{~K} 9 \mathrm{me} 2$ in somatic tissues.

441 The second prediction from our model is that the ectopic inheritance of H3K4

442 methylation at MES-4 germline genes overwhelms the somatic repression systems. Despite

443 the presence of these transcriptional repressor complexes and chromatin remodelers to

444 antagonize MES-4 bookmarking in the soma, loss of spr-5; met-2 maternal reprogramming

445 results in the somatic expression of MES-4 germline genes. This suggests that the failure to

446 add $\mathrm{H} 3 \mathrm{~K} 9 \mathrm{me} 2$, as well as the inappropriate retention of $\mathrm{H} 3 \mathrm{~K} 4 \mathrm{me} 2$, results in a chromatin

447 environment that is permissive for the ectopic maintenance of H3K36me3 in the soma,

448 even in the presence of the systems that repress MES-4 bookmarking somatically (Figure

449 8B). If this is the case, then the developmental delay in spr-5; met-2 progeny should also be

450 dependent upon the activity of the H3K4 methyltransferase. We find that knocking down

451 SET-2, the H3K4me1/2 methyltransferase, rescued the developmental delay that we

452 observe in spr-5; met-2 progeny. These findings suggest that the inheritance of ectopic

453 H3K4 methylation enables the ectopic accumulation of MES-4 dependent H3K36me3, and

454 the subsequent ectopic expression of MES-4 germline genes in somatic tissues of spr-5;

455 met-2 progeny. It is not entirely clear how the subsequent ectopic maintenance of

456 H3K36me3 facilitates ectopic expression. However, it should be noted that there were

457 some differences in which MES-4 germline genes were ectopically expressed between our

458 two spr-5; met-2 RNA-seq experiments. This stochasticity is consistent with H3K36me3

459 being permissive, rather than instructive, for transcription. If this is the case, it is doubtful 
that the spr-5; met-2 developmental delay is caused by the inappropriate expression of any single MES-4 germline gene. Rather, it is likely that the developmental delay is caused by

462 either the inappropriate expression of multiple MES-4 germline genes, or the ectopic 463 activation of the MES-4 germline program.

Conservation of maternal epigenetic reprogramming between invertebrates and

vertebrates organisms must solve (Lee and Katz, 2020). Thus, it is possible that the mechanisms of epigenetic reprogramming may be conserved. Along with the Heard lab, we previously

470 demonstrated that progeny from mice that maternally lack the vertebrate SPR-5 homolog, 471 KDM1A/LSD1, ectopically maintain the expression of germline genes in the embryo. This

472 causes embryonic lethality at the two-cell stage (Ancelin et al., 2016; Wasson et al., 2016).

473 Similarly, maternal loss of the MET-2 ortholog SETDB1 or the MES-4 ortholog NSD1 in mice

474 results in early embryonic lethality (J. Kim et al., 2016; Rayasam et al., 2003). Together

475 these results underscore the developmental importance of properly regulating histone

476 methylation between generations and raise the possibility that the mechanism we have

477 uncovered is conserved in mammals.

$478 \quad$ The model that we have proposed may also help explain the mechanism underlying

479 patients harboring mutations in various histone-modifying enzymes. Recent genome

480 sequencing has revealed that several neurodevelopmental disorders are caused by

481 mutations in histone modifying enzymes (extensively reviewed by (J.-H. Kim et al., 2017).

482 These include mutations in: 1) the H3K36 methyltransferase Setd2 and the H3K27 
483 demethylase Kdm6a, which cause Kabuki Syndrome (Lederer et al., 2012), 2) the human

484 ortholog of $s p r-5$, Lsd1, which causes a Kabuki-like Syndrome (Chong et al., 2016; Tunovic

485 et al., 2014), and 3) the H3K36 methyltransferase Nsd1 which causes Sotos Syndrome

486 (Kurotaki et al., 2002). Similar to what we observed in spr-5; met-2 mutant progeny, many

487 of the human patients with mutations in these histone modifying enzymes suffer from

488 global developmental delay. Based on our model, it is possible that the developmental

489 delay in these patients may be caused by the failure to properly regulate histone

490 methylation during critical developmental transitions. The resulting inappropriate

491 inheritance of histone methylation could result in the ectopic expression of a

492 developmental program in an inappropriate tissue, leading to background noise and

493 developmental delay.

494

495 MATERIALS AND METHODS

496 Strains. All Caenorhabditis elegans strains were grown and maintained at $20^{\circ} \mathrm{C}$ under

497 standard conditions, as previously described (Brenner, 1974). The C. elegans spr-5

498 (by101)(I) strain was provided by R. Baumeister. The N2 Bristol wild-type (WT) strain was

499 provided by the Caenorhabditis Genetics Center. The met-2 (n4256)(III) strain was provided

500 by R. Horvitz. The hT2 [bli-4(e937)let-?(q782)qls48] (I;III) balancer strain was used to

501 maintain spr-5 (by101)(I); met-2 (n4256)(III) double-mutant animals as heterozygotes.

502 Because the $h T 2$ [bli-4(e937)let-?(q782)qls48](I;III) balancer allele does not extend

503 completely to the spr-5 locus on chromosome I, the F0 animals used to generate F1 spr-5;

504 met-2 progeny were cloned out and genotyped to confirm the presence of the $s p r-5$

505 (by101)(I) allele. For genotyping, single animals were picked into 5-10ul of lysis buffer 
(50mM KCl, 10mM Tris-HCl (pH 8.3), 2.5mM MgCl2, 0.45\% NP-40, 0.45\% Tween-20, 0.01\% gelatin) and incubated at $65^{\circ} \mathrm{C}$ for 1 hour followed by $95^{\circ} \mathrm{C}$ for 30 minutes. PCR reactions were performed with AmpliTaq Gold (Invitrogen) according to the manufacturer's protocol and reactions were resolved on agarose gels (see supplementary file 5 for primer sequences). Before completing this study we acquired the FX30208 tmC27 [unc-

512 locus on chromosome I. The qC1 [qls26 (lag2::GFP + rol-6(su1006)](III) strain was obtained 513 from W. Kelly and crossed to met-2 (n4256)(III) to maintain met-2(n4256)(III) as

514 heterozygotes. The spr-5 (by101)(I)/tmC27[unc-75(tmls1239)](I); met-2 (n4256) (III)/qC1 spr-5 (by101)( I); met-2 (n4256)(III) double-mutant animals as balanced heterozygotes. The LET-858::GFP (pha-1(e2123ts)(III); let-858::gfp (ccEx7271)) (Kelly and Fire, 1998)

518 transgenic strain used in somatic transgene silencing assays was acquired from W. Kelly.

519 Scoring developmental delay. C. elegans adult hermaphrodites were allowed to lay

520 embryos for 2-4 hours and then removed in order to synchronize the development of

521 progeny. Progeny were then imaged and scored for development to the adult stage at

522 either 72 hours or seven days after synchronized lay, depending on the experiment.

523 RNA sequencing and analysis. Total RNA was isolated using TRIzol reagent (Invitrogen)

524 from $200-250$ starved L1 larvae born at room temperature $\left(21^{\circ} \mathrm{C}-22^{\circ} \mathrm{C}\right)$ overnight in $\mathrm{M} 9$

525 Buffer. Due to difficulty in isolating large numbers of spr-5; met-2 double-mutant progeny

526 from the $h T 2$ [bli-4(e937)let-?(q782)qls48](I;III) balancer strain, we submitted total RNA to

527 the Genomic Services Laboratory (GSL) (HudsonAlpha, Huntsville, Alabama) for low input

528 RNA-seq services. This service utilizes the Ovation RNA-Seq System V2 kit (Nugen) for 
529 initial RNA amplification prior to library preparation and sequencing (Illumina HiSeq v4,

$53050 \mathrm{bp}$ paired-end reads). For each genotype, 2 biological replicates were obtained. During

531 the course of these experiments, the FX30208 tmC27 [unc-75(tmls1239)](I) balancer

532 became available from the Caenorhabditis Genetics Center. This balancer completely covers

533 the spr-5 locus on chromosome I. Using this balanced strain, we performed a repeat spr-5;

534 met-2 RNA-seq experiment with three additional biological replicates of spr-5; met-2 versus

535 N2 L1 progeny. We submitted the total RNA from new replicates of the repeat RNA-seq to

536 Georgia Genomics and Bioinformatics Core (University of Georgia, Athens, Georgia) for

537 standard Poly-A RNA-seq services (Illumina Nextseq, 50bp paired-end reads). Downstream

538 quality control and analysis were performed identically for both RNA-seq experiments. For

539 both the low-input and repeat standard RNA-seq, sequencing reads were checked for

540 quality using FastQC (Wingett and Andrews, 2018), filtered using Trimmomatic (Bolger et

541 al., 2014), and remapped to the C. elegans transcriptome (ce10, WS220) using HISAT2 (D.

542 Kim et al., 2015). Read count by gene was obtained by FeatureCounts (Liao et al., 2014).

543 Differentially expressed transcripts for the low-input RNA-seq experiment (significance

544 threshold, Wald test, p-value $<0.05$ ) and the repeat RNA-seq experiment (significance

545 threshold, Wald test, $\mathrm{p}$-adj < 0.05) were determined using DESEQ2 (v.2.11.40.2) (Love et

546 al., 2014). Transcripts per million (TPM) values were calculated from raw data obtained

547 from FeatureCounts output. Subsequent downstream analysis was performed using R with

548 normalized counts and p-values from DESEQ2 (v.2.11.40.2). Heatmaps were produced

549 using the ComplexHeatmap R Package (Gu et al., 2016). Data was scaled and hierarchical

550 clustering was performed using the complete linkage algorithm. In the linkage algorithm,

551 distance was measured by calculating pairwise distance. Volcano plots were produced 
552 using the EnhancedVolcano package (v.0.99.16). Additionally, Gene Ontology (G0) Pathway

553 analysis was performed using the online platform WormEnrichr (Chen et al., 2013;

554 Kuleshov et al., 2016). Rechtsteiner and colleagues identified 214 MES-4 germline genes

555 (Rechtsteiner et al., 2010). 17 of these genes are pseudogenes that we were unable to

556 convert from Ensembl transcript IDs to RefSeq mRNA accession, and another gene was

557 duplicated, so we removed those genes. This leaves 196 MES-4 germline genes that we

558 used for our analysis. An additional heatmap comparison of differentially expressed genes

559 between spr-5, met-2, and spr-5; met-2 progeny compared to N2 progeny was generated in

560 Microsoft Excel using log2 fold change values from the DESEQ2 analysis. Because transcript

561 isoforms were ignored, we discuss the data in terms of "genes expressed" rather than

562 "transcripts expressed".

563 Chromatin immunoprecipitation sequencing and analysis. Chromatin

564 immunoprecipitation (ChIP) experiments were performed as described by Katz and

565 colleagues (Katz et al., 2009). Briefly, 600 starved L1 larvae born at room temperature

$566\left(21^{\circ} \mathrm{C}-22^{\circ} \mathrm{C}\right)$ overnight in $\mathrm{M} 9$ Buffer were collected, frozen in liquid nitrogen, and stored at

$567-80^{\circ} \mathrm{C}$ prior to homogenization. Frozen pellets were disrupted by a glass Dounce

568 homogenizer, fixed with formaldehyde (1\% final concentration), and quenched with

569 glycine. ChIP samples were processed with a Chromatin Immunoprecipitation Assay Kit

570 (Millipore), according to manufacturer's instructions. Samples were sonicated using a

571 Diagenode Bioruptor UCD-200 at $4^{\circ} \mathrm{C}$ on the "high" setting for a total of $30 \mathrm{~min}$ with a cycle

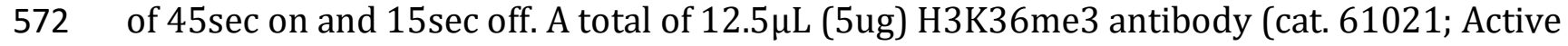

573 motif) was used for immunoprecipitation. The Genomic Services Laboratory (GSL)

574 (HudsonAlpha, Huntsville, Alabama) performed library preparation and sequencing 
575 (Illumina HiSeq v4, 50bp single-end reads). Reads were checked for quality using FastQC

576 (Wingett and Andrews, 2018) and remapped to the C. elegans transcriptome (ce10, WS220)

577 using Bowtie2 (Langmead and Salzberg, 2012; Langmead et al., 2009) under default

578 parameters. bamCoverage in deepTools2 (Ramírez et al., 2016) was used to generate

579 bigwig coverage tracks in 50bp bins, with blacklisted regions from McMurchy et al. 2017

excluded, using the following parameters: -bs 50, --normalizeUsing RPKM (McMurchy et al.,

581 2017). MACS2 (Feng et al., 2012; Zhang et al., 2008) default parameters were used to call

582 peaks and create bedgraph files for sequenced and mapped H3K36me3 ChIP samples and

583 input DNA samples with the following adjustments to account for H3K36me3 broader

584 domains: Broad-cutoff $=0.001$. Blacklisted regions from McMurchy et al. 2017 were

585 excluded for this analysis (McMurchy et al., 2017). Using published H3K36me3 modMine

586 Chip-chip called broad peaks (modENCODE_3555) from N2 L1 larvae as a guide, we then

587 merged called broad peaks within 1200bp using Bedtools: MergeBED (Quinlan and Hall,

588 2010). H3K9me2 bedgraph files used in our analysis were from a published dataset

589 (Rechtsteiner et al., 2019). Integrative Genome Viewer (IGV) was used to visualize

590 H3K36me3 reads normalized to reads per kilobase millions (RPKM) and H3K9me2 reads

591 normalized to 15 million reads (genome wide coverage of H3K9me2; (Rechtsteiner et al.,

592 2019).

593 RNAi methods. RNAi by feeding was carried out using clones from the Ahringer library

594 (Kamath and Ahringer, 2003). Feeding experiments were performed on RNAi plates (NGM

595 plates containing $100 \mathrm{ug} / \mathrm{ml}$ ampicillin, $0.4 \mathrm{mM}$ IPTG, and 12.5ug/ml tetracycline). F0

596 worms were placed on RNAi plates as L2 larvae and then moved to fresh RNAi plates 48hrs

597 later where they were allowed to lay embryos for 2-4 hrs. F0 worms were then removed 
598 from plates and sacrificed or placed in M9 buffer overnight so that starved L1 progeny

599 could be isolated for quantitative PCR (qPCR). F1 progeny were scored 72hrs after the

600 synchronized lay for developmental progression. For each RNAi experiment, pos-1 RNAi

601 was used as a positive control. Each RNAi experiment reported here pos-1 RNAi resulted in

$602>95 \%$ embryonic lethality, indicating that RNAi plates were optimal.

603 Real-time expression analysis. Total RNA was isolated using TRIzol reagent (Invitrogen)

604 from synchronized L1s born at room temperature $\left(21^{\circ} \mathrm{C}-22^{\circ} \mathrm{C}\right)$. cDNA synthesis and qPCR

605 were carried out as described (Kerr et al. 2014). A total of two biological replicates were

606 performed and for both biological replicates experiments were performed in triplicate and

607 normalized to ama-1 mRNA expression (see supplementary file 5 for primer sequences).

608 Differential interference contrast microscopy. Worms were immobilized in $0.1 \%$

609 levamisole and placed on a 2\% agarose pad for imaging at either 10x or 40x magnification.

$61040 x$ DIC images were overlaid together using Adobe photoshop to generate high resolution

611 images of whole worms.

612 Single Molecule Fluorescent in situ Hybridization (smFISH). Quasar 570 labeled

613 smFISH probe sets for $h t p-1$ and $c p b-1$ were designed using Stellaris Probe Designer

614 (Biosearch) (see supplementary file 4 for probe sequences). The $h t p-1 \mathrm{smFISH}$ probes were

615 designed using the complete 1,059nt $h t p$-1 protein-coding sequence. Likewise, The $c p b-1$

616 smFISH probes were designed using the complete 1,683nt $c p b$-1 protein-coding sequence.

617 In addition, an smFISH fluorescent probe set for ama-1 was purchased from the

618 DesignReady catalog (cat\#: VSMF-6002-5, Biosearch). Synchronized L1 larvae for smFISH

619 were obtained by bleaching 300-500 gravid hermaphrodites and allowing embryos to

620 hatch overnight on $6 \mathrm{~cm}$ NGM plates lightly seeded OP50 bacteria. L1 larvae were then 
621 washed into 1.5ul Eppendorf tubes using nuclease-free M9 buffer. Fixation and

622 hybridization steps followed the Stellaris RNA FISH protocol for C. elegans adapted from

623 RAJ lab protocol (Raj and Tyagi, 2010). In brief, we resuspended L1 larvae in fixation buffer

624 (3.7\% formaldehyde in $1 \times$ PBS) for 15 minutes at room temperature then transferred

625 tubes to liquid nitrogen. Samples were thawed in water and placed on ice for 20 minutes. In

626 our hands, we obtain better fluorescent signal by freeze cracking L1 larvae. Following

627 fixation, L1 Larvae were resuspended in $70 \% \mathrm{EtOH}$ and stored at $4^{\circ} \mathrm{C}$ for $24-48$ hours. For

628 all probe sets, we incubated L1 larvae in 100ul hybridization buffer (containing 10\%

629 formamide and $125 \mathrm{~nm}$ probe) for 4 hours at $37^{\circ} \mathrm{C}$. After hybridization, samples were

630 washed in wash buffer at $37^{\circ} \mathrm{C}$ for 30 minutes, incubated in $50 \mathrm{ng} / \mathrm{mL}$ DAPI in wash buffer

631 at $30^{\circ} \mathrm{C}$ for 30 minutes, washed once in $2 x$ SSC for 2 minutes at room temperature, and

632 mounted in Vectashield mounting medium. Mounted slides were imaged immediately using

633 a 100x objective on a spinning-disk confocal Nikon-TiE imaging system. Images were

634 captured using NIS-Elements software (Nikon) and ImageJ (NIH, http://imagej.nih.gov/ij/)

635 was used for viewing. Image J maximum projection was used to project z-stack images to a

636 single plane. The fluorescent intensity of smFISH dots were $>2$-fold above background as

637 expected (Ji and van Oudenaarden, 2012).

638 LET-858::GFP transgene silencing assay. First generation spr-5; met-2 hermaphrodites

639 were crossed to let-858 transgenic males to generate spr-5/+; met-2/+; let-858::gfp animals.

640 The let-858 transgene is an extrachromosomal multicopy let-858 array (Kelly and Fire,

641 1998). From these animals we generated spr-5; met-2; let-858::gfp animals and scored them

642 for somatic expression of LET-858::GFP using a standard stereoscope. L2 progeny from N2

643 and spr-5; met-2 progeny expressing the LET-858::GFP transgene were scored as "bright" 
644 (High level ubiquitous expression), faint (barely visible and ubiquitous expression), or off

645 (no expression). Because less than $50 \%$ of progeny inherit the let-858 transgene, we

646 normalized the percentage of progeny scored as "off" for LET-858::GFP to presence of the

647 let-858 transgene based on genotyping for $g f p$. For N2, 0 out of 60 progeny that were

648 scored as "off" failed to inherit the let-858 transgene indicating that the transgene is never

649 silenced in N2 progeny (data not shown, see supplementary file 5 for primer sequences).

650 For spr-5; met-2 progeny, 8 out of 50 progeny that were scored as "off" inherited the let-858

651 transgene indicating that the let-858 transgene is completely silenced in some spr-5; met-2

652 progeny (data not shown).

653

\section{ACKNOWLEDGEMENTS}

655 We are grateful to members of the Katz lab, as well as T. Caspary and C. Bean, for their

656 helpful discussion and critical reading of the manuscript; M. J. Rowley for advice on

657 bioinformatics; S. Strome and L. Petrella for helpful discussion and experimental advice; D.

658 Lerit, R. Deal, V. Corces, and W. Kelly for the generous sharing of reagents and equipment.

659 We thank R. Horvitz, W. Kelly, and the Caenorhabditis Genetics Center (funded by NIH P40

660 OD010440) for strains. Funding: This work was funded by a grant to D.J.K. (NSF

661 IOS1931697); T.W.L. and B.S.C. were supported by the Fellowships in Research and Science

662 Teaching IRACDA postdoctoral program (NIH K12GM00680-15); and B.S.C. was also

663 supported by NIH F32 GM126734-01. Author Contributions: B.S.C. and D.J.K. conceived

664 and designed the study and wrote the manuscript. B.S.C. performed experiments under the

665 direction of D.J.K. B.S.C and D.J.K. analyzed data and interpreted results, with help from

666 T.W.L. J.S.B. initially performed set-2 RNAi rescue experiment and C.F.P. initially performed 
667

668

669

670

671

672

673

674

675

676

677

678

679

680

681

682

683

684

685

686

687

688

689

690

691

692

693

694

695

696

697

the let-858 transgene silencing experiment. D.A.M. helped with RNA-seq analysis. All authors discussed the results. Data availability: Raw and processed genomic data has been deposited with the Gene Expression Omnibus (www.ncbi.nlm.nih.gov/geo) under accession code GSE143839.

\section{Supplementary Material}

Figure Supplements:

Figure 1-figure supplement 1

Figure 2-figure supplements 1, 2, and 3

Figure 3-figure supplement 1

Figure 4-figure supplements 1 and 2

Supplementary files 1-6

Source Data files 1-3

\section{REFERENCES}

Ancelin, K., Syx, L., Borensztein, M., Ranisavljevic, N., Vassilev, I., Briseño-Roa, L., Liu, T., Metzger, E., Servant, N., Barillot, E., Chen, C.-J., Schüle, R., Heard, E., 2016. Maternal LSD1/KDM1A is an essential regulator of chromatin and transcription landscapes during zygotic genome activation. Elife 5, 4615. doi:10.7554/eLife.08851

Batchelder, C., Dunn, M.A., Choy, B., Suh, Y., Cassie, C., Shim, E.Y., Shin, T.H., Mello, C., Seydoux, G., Blackwell, T.K., 1999. Transcriptional repression by the Caenorhabditis elegans germ-line protein PIE-1. Genes Dev. 13, 202-212. doi:10.1101/gad.13.2.202

Bolger, A.M., Lohse, M., Usadel, B., 2014. Trimmomatic: a flexible trimmer for Illumina sequence data. Bioinformatics 30, 2114-2120. doi:10.1093/bioinformatics/btu170

Brenner, S., 1974. The genetics of Caenorhabditis elegans. Genetics 77, 71-94.

Buckley, B.A., Burkhart, K.B., Gu, S.G., Spracklin, G., Kershner, A., Fritz, H., Kimble, J., Fire, A., Kennedy, S., 2012. A nuclear Argonaute promotes multigenerational epigenetic inheritance and germline immortality. Nature 489, 447-451. doi:10.1038/nature11352

Capowski, E.E., Martin, P., Garvin, C., Strome, S., 1991. Identification of grandchildless loci whose products are required for normal germ-line development in the nematode Caenorhabditis elegans. Genetics 129, 1061-1072. 
Chen, E.Y., Tan, C.M., Kou, Y., Duan, Q., Wang, Z., Meirelles, G.V., Clark, N.R., Ma'ayan, A., 2013. Enrichr: interactive and collaborative HTML5 gene list enrichment analysis tool. BMC Bioinformatics 14, 128. doi:10.1186/1471-2105-14-128

Chong, J.X., Yu, J.-H., Lorentzen, P., Park, K.M., Jamal, S.M., Tabor, H.K., Rauch, A., Saenz, M.S., Boltshauser, E., Patterson, K.E., Nickerson, D.A., Bamshad, M.J., 2016. Gene discovery for Mendelian conditions via social networking: de novo variants in KDM1A cause developmental delay and distinctive facial features. Genet. Med. 18, 788-795. doi:10.1038/gim.2015.161

Feng, J., Liu, T., Qin, B., Zhang, Y., Liu, X.S., 2012. Identifying ChIP-seq enrichment using MACS. Nat Protoc 7, 1728-1740. doi:10.1038/nprot.2012.101

Fukuyama, M., Rougvie, A.E., Rothman, J.H., 2006. C. elegans DAF-18/PTEN mediates nutrient-dependent arrest of cell cycle and growth in the germline. Curr. Biol. 16, 773779. doi:10.1016/j.cub.2006.02.073

Furuhashi, H., Takasaki, T., Rechtsteiner, A., Li, T., Kimura, H., Checchi, P.M., Strome, S., Kelly, W.G., 2010. Trans-generational epigenetic regulation of C. elegans primordial germ cells. Epigenetics Chromatin 3, 15. doi:10.1186/1756-8935-3-15

Garvin, C., Holdeman, R., Strome, S., 1998. The phen otype of mes-2, mes-3, mes-4 and mes6 , maternal-effect genes required for survival of the germline in Caenorhabditis elegans, is sensitive to chromosome dosage. Genetics 148, 167-185.

$\mathrm{Gu}, \mathrm{Z}$., Eils, R., Schlesner, M., 2016. Complex heatmaps reveal patterns and correlations in multidimensional genomic data. Bioinformatics 32, 2847-2849. doi:10.1093/bioinformatics/btw313

Jambhekar, A., Dhall, A., Shi, Y., 2019. Roles and regulation of histone methylation in animal development. Nat. Rev. Mol. Cell Biol. 20, 625-641. doi:10.1038/s41580-019-0151-1

Ji, N., van Oudenaarden, A., 2012. Single molecule fluorescent in situ hybridization (smFISH) of C. elegans worms and embryos. WormBook 1-16. doi:10.1895/wormbook.1.153.1

Kamath, R.S., Ahringer, J., 2003. Genome-wide RNAi screening in Caenorhabditis elegans. Methods 30, 313-321. doi:10.1016/s1046-2023(03)00050-1

Katz, D.J., Edwards, T.M., Reinke, V., Kelly, W.G., 2009. A C. elegans LSD1 demethylase contributes to germline immortality by reprogramming epigenetic memory. Cell 137, 308-320. doi:10.1016/j.cell.2009.02.015

Kelly, W.G., Fire, A., 1998. Chromatin silencing and the maintenance of a functional germline in Caenorhabditis elegans. Development 125, 2451-2456.

Kerr, S.C., Ruppersburg, C.C., Francis, J.W., Katz, D.J., 2014. SPR-5 and MET-2 function cooperatively to reestablish an epigenetic ground state during passage through the germ line. Proc. Natl. Acad. Sci. U.S.A. 111, 9509-9514. doi:10.1073/pnas.1321843111

Kim, D., Langmead, B., Salzberg, S.L., 2015. HISAT: a fast spliced aligner with low memory requirements. Nat. Methods 12, 357-360. doi:10.1038/nmeth.3317

Kim, J., Zhao, H., Dan, J., Kim, S., Hardikar, S., Hollowell, D., Lin, K., Lu, Y., Takata, Y., Shen, J., Chen, T., 2016. Maternal Setdb1 Is Required for Meiotic Progression and Preimplantation Development in Mouse. PLoS Genet. 12, e1005970. doi:10.1371/journal.pgen.1005970

Kim, J.-H., Lee, J.H., Lee, I.-S., Lee, S.B., Cho, K.S., 2017. Histone Lysine Methylation and Neurodevelopmental Disorders. Int J Mol Sci 18, 1404. doi:10.3390/ijms18071404 
Kreher, J., Takasaki, T., Cockrum, C., Sidoli, S., Garcia, B.A., Jensen, O.N., Strome, S., 2018. Distinct Roles of Two Histone Methyltransferases in Transmitting H3K36me3-Based Epigenetic Memory Across Generations in Caenorhabditis elegans. Genetics 210, 969982. doi:10.1534/genetics.118.301353

Kuleshov, M.V., Jones, M.R., Rouillard, A.D., Fernandez, N.F., Duan, Q., Wang, Z., Koplev, S., Jenkins, S.L., Jagodnik, K.M., Lachmann, A., McDermott, M.G., Monteiro, C.D., Gundersen, G.W., Ma'ayan, A., 2016. Enrichr: a comprehensive gene set enrichment analysis web server 2016 update. Nucleic Acids Res. 44, W90-7. doi:10.1093/nar/gkw377

Kurotaki, N., Imaizumi, K., Harada, N., Masuno, M., Kondoh, T., Nagai, T., Ohashi, H., Naritomi, K., Tsukahara, M., Makita, Y., Sugimoto, T., Sonoda, T., Hasegawa, T., Chinen, Y., Tomita Ha, H.-A., Kinoshita, A., Mizuguchi, T., Yoshiura Ki, K.-I., Ohta, T., Kishino, T., Fukushima, Y., Niikawa, N., Matsumoto, N., 2002. Haploinsufficiency of NSD1 causes Sotos syndrome. Nat. Genet. 30, 365-366. doi:10.1038/ng863

Langmead, B., Salzberg, S.L., 2012. Fast gapped-read alignment with Bowtie 2. Nat. Methods 9, 357-359. doi:10.1038/nmeth.1923

Langmead, B., Trapnell, C., Pop, M., Salzberg, S.L., 2009. Ultrafast and memory-efficient alignment of short DNA sequences to the human genome. Genome Biol. 10, R25. doi:10.1186/gb-2009-10-3-r25

Lederer, D., Grisart, B., Digilio, M.C., Benoit, V., Crespin, M., Ghariani, S.C., Maystadt, I., Dallapiccola, B., Verellen-Dumoulin, C., 2012. Deletion of KDM6A, a histone demethylase interacting with MLL2, in three patients with Kabuki syndrome. Am. J. Hum. Genet. 90, 119-124. doi:10.1016/j.ajhg.2011.11.021

Lee, T.W., Katz, D.J., 2020. Hansel, Gretel, and the Consequences of Failing to Remove Histone Methylation Breadcrumbs. Trends Genet. 36, 160-176. doi:10.1016/j.tig.2019.12.004

Lev, I., Seroussi, U., Gingold, H., Bril, R., Anava, S., Rechavi, O., 2017. MET-2-Dependent H3K9 Methylation Suppresses Transgenerational Small RNA Inheritance. Curr. Biol. 27, 1138-1147. doi:10.1016/j.cub.2017.03.008

Liao, Y., Smyth, G.K., Shi, W., 2014. featureCounts: an efficient general purpose program for assigning sequence reads to genomic features. Bioinformatics 30, 923-930. doi:10.1093/bioinformatics/btt656

Love, M.I., Huber, W., Anders, S., 2014. Moderated estimation of fold change and dispersion for RNA-seq data with DESeq2. Genome Biol. 15, 550. doi:10.1186/s13059-014-0550-8 McMurchy, A.N., Stempor, P., Gaarenstroom, T., Wysolmerski, B., Dong, Y., Aussianikava, D., Appert, A., Huang, N., Kolasinska-Zwierz, P., Sapetschnig, A., Miska, E.A., Ahringer, J., 2017. A team of heterochromatin factors collaborates with small RNA pathways to combat repetitive elements and germline stress. Elife 6, 7931. doi:10.7554/eLife.21666

Mello, C.C., Draper, B.W., Krause, M., Weintraub, H., Priess, J.R., 1992. The pie-1 and mex-1 genes and maternal control of blastomere identity in early C. elegans embryos. Cell 70, 163-176. doi:10.1016/0092-8674(92)90542-k

Petrella, L.N., Wang, W., Spike, C.A., Rechtsteiner, A., Reinke, V., Strome, S., 2011. synMuv B proteins antagonize germline fate in the intestine and ensure C. elegans survival. Development 138, 1069-1079. doi:10.1242/dev.059501

Quinlan, A.R., Hall, I.M., 2010. BEDTools: a flexible suite of utilities for comparing genomic features. Bioinformatics 26, 841-842. doi:10.1093/bioinformatics/btq033 
Raj, A., Tyagi, S., 2010. Detection of individual endogenous RNA transcripts in situ using multiple singly labeled probes. Meth. Enzymol. 472, 365-386. doi:10.1016/S0076$6879(10) 72004-8$

Ramírez, F., Ryan, D.P., Grüning, B., Bhardwaj, V., Kilpert, F., Richter, A.S., Heyne, S., Dündar, F., Manke, T., 2016. deepTools2: a next generation web server for deep-sequencing data analysis. Nucleic Acids Res. 44, W160-5. doi:10.1093/nar/gkw257

Rayasam, G.V., Wendling, O., Angrand, P.-O., Mark, M., Niederreither, K., Song, L., Lerouge, T., Hager, G.L., Chambon, P., Losson, R., 2003. NSD1 is essential for early post-implantation development and has a catalytically active SET domain. EMBO J. 22, 3153-3163. doi:10.1093/emboj/cdg288

Rechtsteiner, A., Costello, M.E., Egelhofer, T.A., Garrigues, J.M., Strome, S., Petrella, L.N., 2019. Repression of Germline Genes in Caenorhabditis elegans Somatic Tissues by H3K9 Dimethylation of Their Promoters. Genetics 212, 125-140. doi:10.1534/genetics.118.301878

Rechtsteiner, A., Ercan, S., Takasaki, T., Phippen, T.M., Egelhofer, T.A., Wang, W., Kimura, H., Lieb, J.D., Strome, S., 2010. The histone H3K36 methyltransferase MES-4 acts epigenetically to transmit the memory of germline gene expression to progeny. PLoS Genet. 6, e1001091. doi:10.1371/journal.pgen.1001091

Seydoux, G., Mello, C.C., Pettitt, J., Wood, W.B., Priess, J.R., Fire, A., 1996. Repression of gene expression in the embryonic germ lineage of C. elegans. Nature 382, 713-716. doi:10.1038/382713a0

Shi, Y., Lan, F., Matson, C., Mulligan, P., Whetstine, J.R., Cole, P.A., Casero, R.A., Shi, Y., 2004. Histone demethylation mediated by the nuclear amine oxidase homolog LSD1. Cell 119, 941-953. doi:10.1016/j.cell.2004.12.012

Shi, Y.-J., Matson, C., Lan, F., Iwase, S., Baba, T., Shi, Y., 2005. Regulation of LSD1 histone demethylase activity by its associated factors. Mol. Cell 19, 857-864. doi:10.1016/j.molcel.2005.08.027

Sijen, T., Fleenor, J., Simmer, F., Thijssen, K.L., Parrish, S., Timmons, L., Plasterk, R.H., Fire, A., 2001. On the role of RNA amplification in dsRNA-triggered gene silencing. Cell 107, 465-476. doi:10.1016/s0092-8674(01)00576-1

Strome, S., 2005. Specification of the germ line. WormBook 1-10. doi:10.1895/wormbook.1.9.1

Sulston, J.E., Schierenberg, E., White, J.G., Thomson, J.N., 1983. The embryonic cell lineage of the nematode Caenorhabditis elegans. Dev. Biol. 100, 64-119. doi:10.1016/00121606(83)90201-4

Tijsterman, M., Okihara, K.L., Thijssen, K., Plasterk, R.H.A., 2002. PPW-1, a PAZ/PIWI protein required for efficient germline $\mathrm{RNAi}$, is defective in a natural isolate of $\mathrm{C}$. elegans. Curr. Biol. 12, 1535-1540. doi:10.1016/s0960-9822(02)01110-7

Tunovic, S., Barkovich, J., Sherr, E.H., Slavotinek, A.M., 2014. De novo ANKRD11 and KDM1A gene mutations in a male with features of KBG syndrome and Kabuki syndrome. Am. J. Med. Genet. A 164A, 1744-1749. doi:10.1002/ajmg.a.36450

Unhavaithaya, Y., Shin, T.H., Miliaras, N., Lee, J., Oyama, T., Mello, C.C., 2002. MEP-1 and a homolog of the NURD complex component Mi-2 act together to maintain germlinesoma distinctions in C. elegans. Cell 111, 991-1002. 
832 Wang, D., Kennedy, S., Conte, D., Kim, J.K., Gabel, H.W., Kamath, R.S., Mello, C.C., Ruvkun, G., 833 2005. Somatic misexpression of germline P granules and enhanced RNA interference in retinoblastoma pathway mutants. Nature 436, 593-597. doi:10.1038/nature04010 D.J., 2016. Maternally provided LSD1/KDM1A enables the maternal-to-zygotic transition and prevents defects that manifest postnatally. Elife 5, 296. doi:10.7554/eLife.08848

Wingett, S.W., Andrews, S., 2018. FastQ Screen: A tool for multi-genome mapping and quality control. F1000Res 7, 1338. doi:10.12688/f1000research.15931.2 Myers, R.M., Brown, M., Li, W., Liu, X.S., 2008. Model-based analysis of ChIP-Seq (MACS). Genome Biol. 9, R137. doi:10.1186/gb-2008-9-9-r137 
Figure 1. spr-5; met-2 mutants display severe developmental delay. 10x Differential Interference Contrast (DIC) images of N2 (A), spr-5 (B), met-2 (C), and spr-5; met-2 progeny (D) 72 hours post synchronized lay. Scale bar: $100 \mu \mathrm{m}$. (E) Percentage of N2, spr-5, met-2, and spr-5; met-2 progeny that reached the adult stage (\% Adult Progeny) by 72 hours post synchronized lay. Error bars represent the standard deviation of the mean from three experiments. $\mathrm{N}=$ the total number of progeny 20-25 hermaphrodites scored over three experiments.

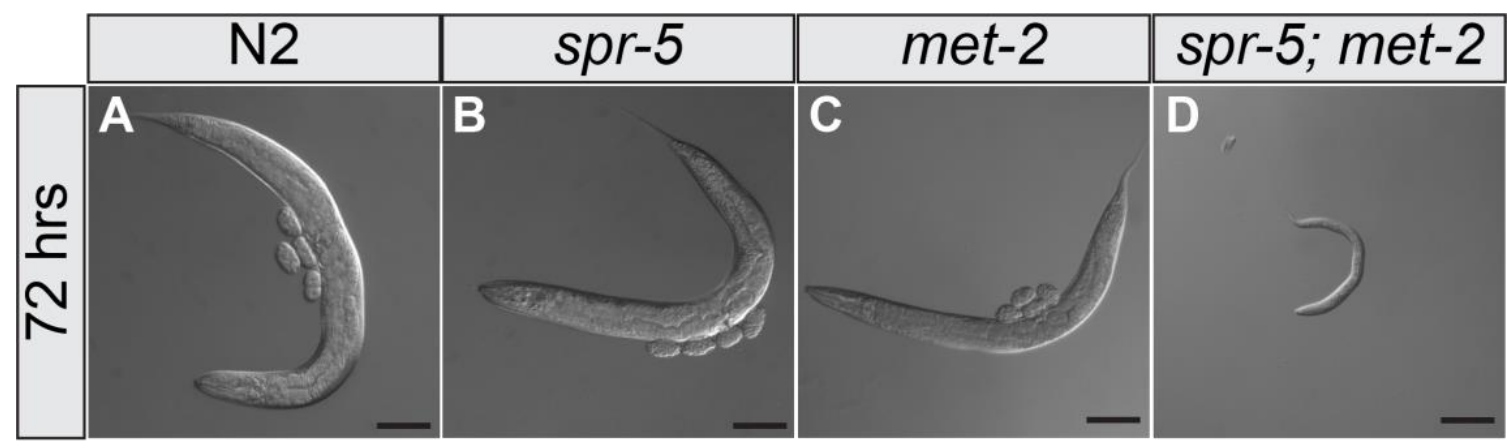

$\mathbf{E}$

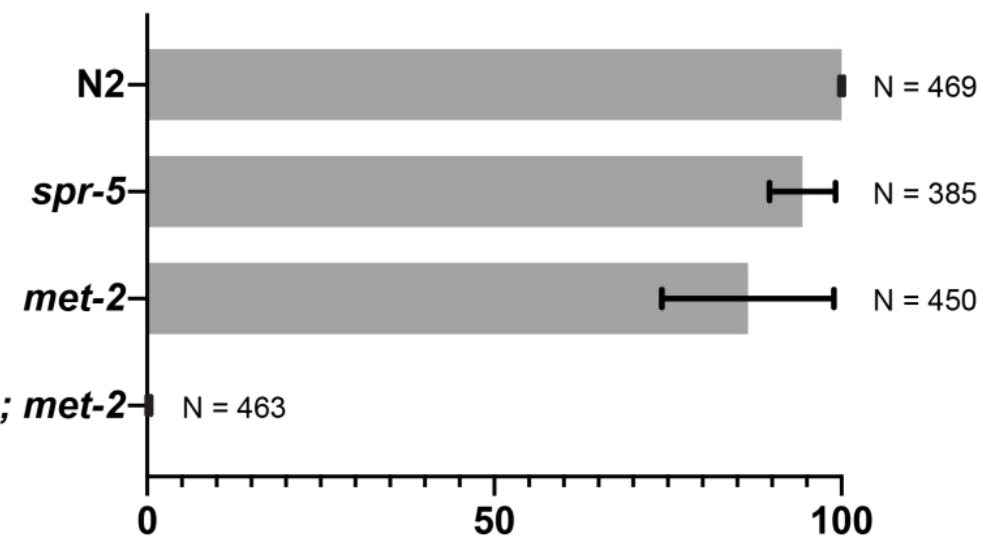

$\%$ Adult Progeny 
Figure 2. MES-4 germline genes are ectopically expressed in spr-5; met-2 mutant soma. Overlap between MES-4 germline genes and genes up-regulated (A) and downregulated (B) in spr-5; met-2 L1 progeny. Significant over-enrichment in A was determined by the hypergeometric test ( ${ }^{*} \mathrm{p}$-value $<6.44 \mathrm{e}-20$ ). (C) Heatmap of log2 fold change (FC) of 176 MES-4 germline genes in spr-5, met-2 and spr-5; met-2 mutants compared to N2. (D) Heatmap of $\log 2 \mathrm{FC}$ of 176 MES-4 germline genes for the repeat spr-5; met-2 RNA-seq experiment, with three additional biological replicates of spr-5; met-2* mutants compared to $\mathrm{N} 2$ (see methods). Log2FC values are represented in a yellow to blue gradient and range from -2 to 5 . Yellow represents genes with negative log2FC values and blue represents genes with positive log2FC values compared to N2. The remaining 21 MES-4 germline genes were not included because they do not have an expression value in one or more of the data sets (spr-5, met-2, or spr-5; met-2).
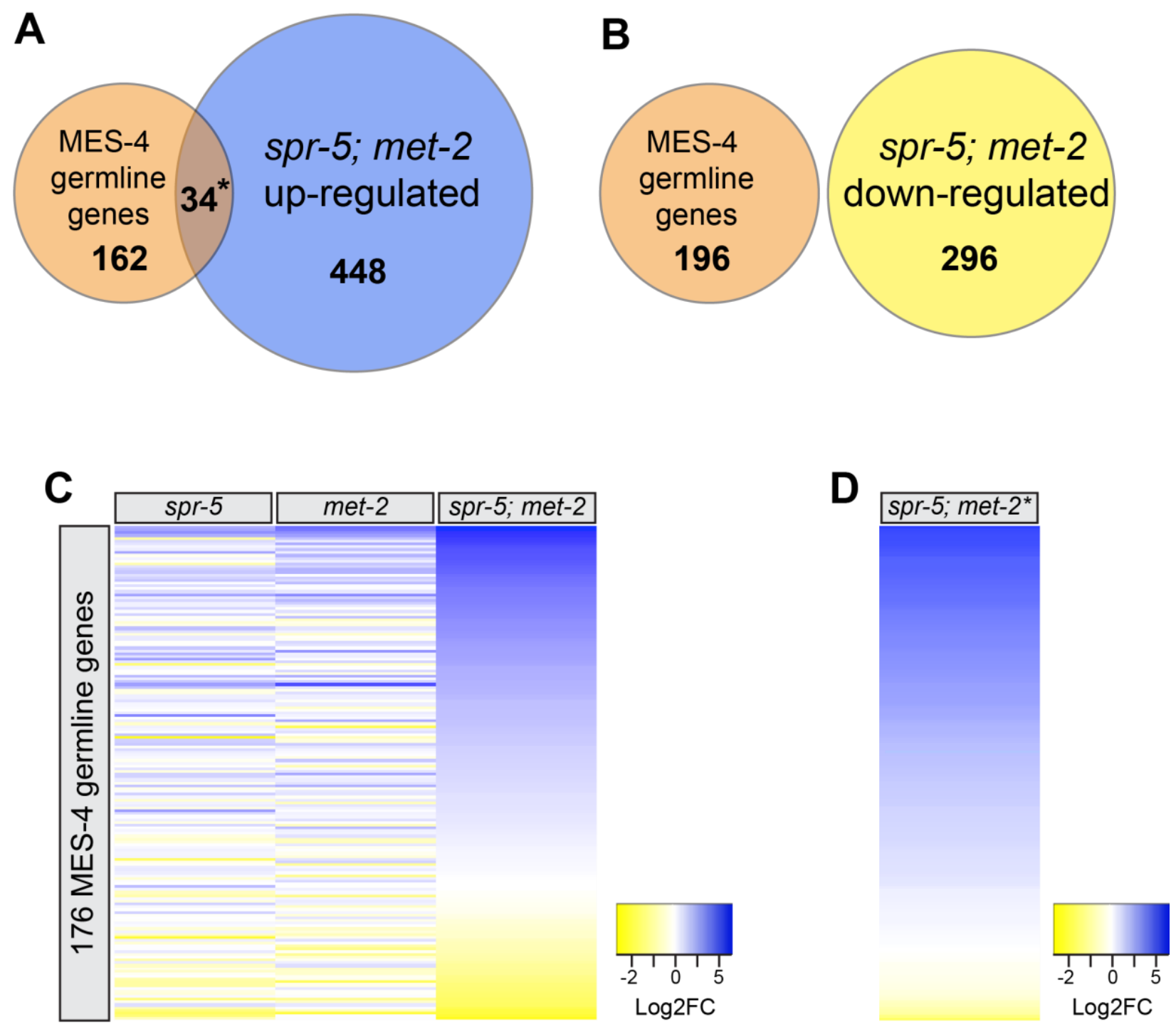
bioRxiv preprint doi: https://doi.org/10.1101/2020.01.22.914580; this version posted May 29, 2020. The copyright holder for this preprint (which

was not certified by peer review) is the author/funder, who has granted bioRxiv a license to display the preprint in perpetuity. It is made available under aCC-BY-NC-ND 4.0 International license.

Figure 3. spr-5; met-2 L1 progeny ectopically express MES-4 germline genes in multiple somatic tissues. 40x smFISH images of $h t p-1$ (A, C, D, F) and $c p b-1$ (G, I, J, L) endogenous mRNAs in N2 (A-C, G-I) and spr-5; met-2 (D-F, J-L) L1 progeny. DAPI was used as a nuclear marker (B, C, E, F, H, I, K, L). Insets are high magnification images of the germ cells, Z2 and Z3, in N2 L1 progeny. Arrows (J, L) denote ectopic cpb-1 mRNA foci in somatic cells. Scale bar $40 \mu \mathrm{m}$.
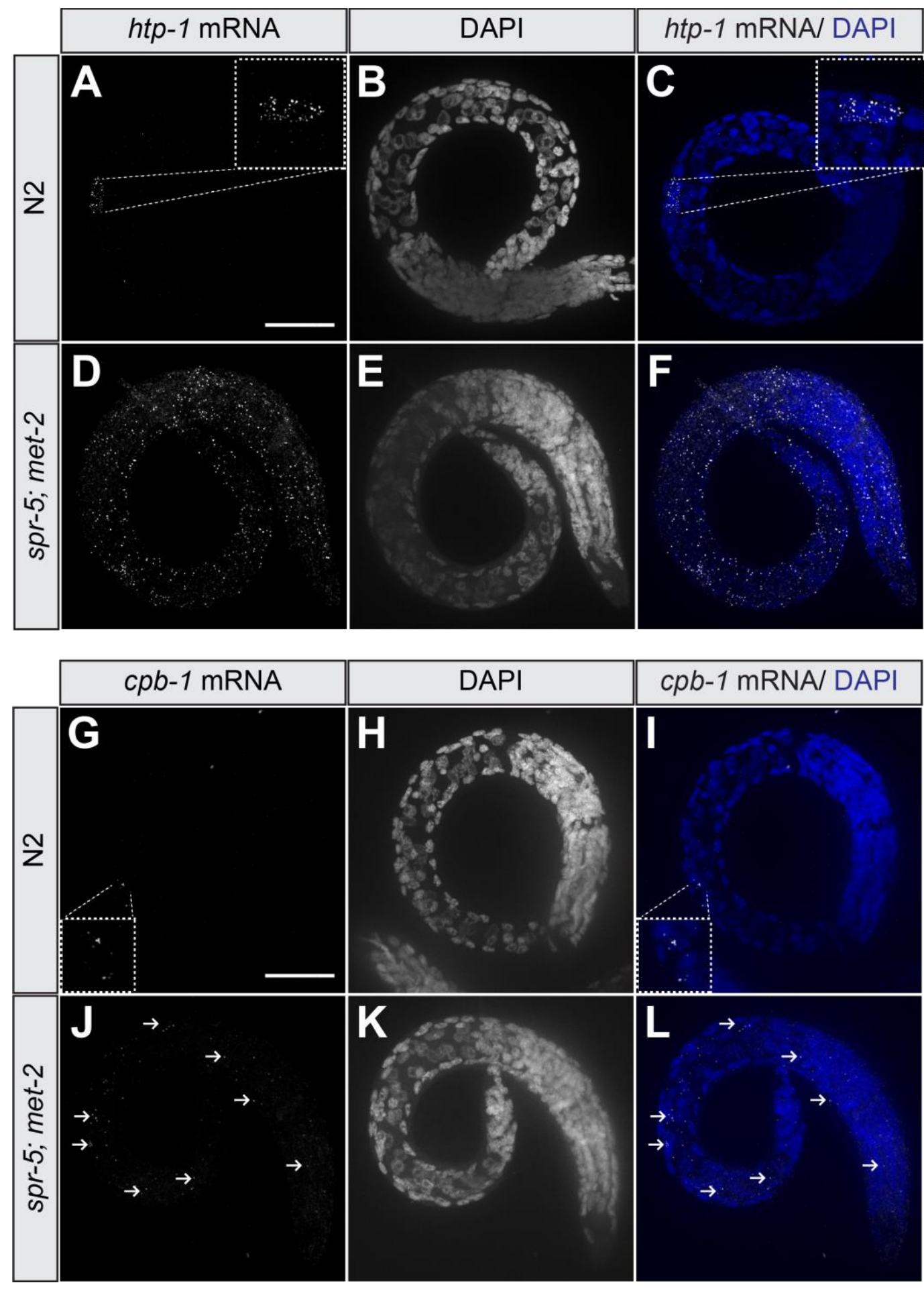
Figure 4. MES-4 germline genes display ectopic H3K36me3 in spr-5; met-2 L1 progeny. Heatmap of H3K36me3 ChIP-seq reads normalized to reads per kilobase million (RPKM) over the gene bodies of 196 MES-4 germline genes in wild-type (N2) (A) versus spr-5; met-2 (B) L1 progeny (second replicate in Figure S5). Gene bodies were pseudoscaled to $1 \mathrm{~kb}$ with $500 \mathrm{bp}$ borders separated by orange bars that represent the transcriptional start site (TSS) and transcriptional end site (TES). Integrative Genome Viewer (IGV) image of H3K36me3 ChIP-seq reads normalized to RPKM at MES-4 germline genes (C-F, J-M) and control genes (G-I, N-P) in N2 (C-I) versus spr-5; met-2 (J-P) L1 progeny. RPKM IGV windows were scaled between 0 and 202 RPKM for all genes.
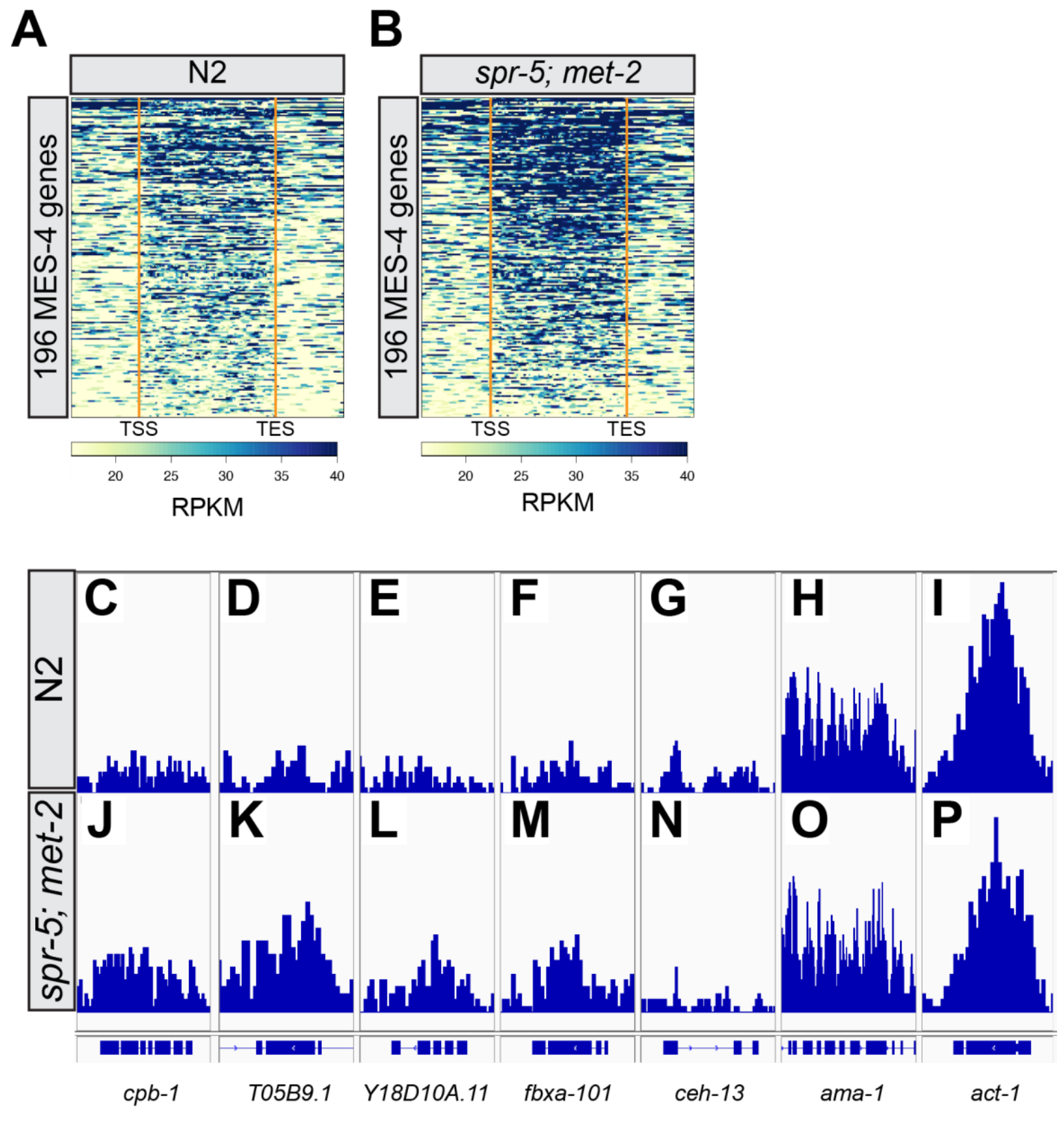
Figure 5. Knocking down MES-4 rescues ectopic expression of MES-4 germline genes in spr-5; met-2 L1 progeny. Quantitative RT-PCR showing the relative units of expression for nine MES-4 germline genes (cpb-1, csr-1, fbxa-101, htp-1, T05B9.1, Y18D10A.11, ftr-1, rmh-1, K12D12.5) in L1 progeny of spr-5; met-2 hermaphrodites fed either control L4440 RNAi (grey bars) or mes-4 RNAi (black bars) versus N2 fed control L4440 RNAi (white bars). Relative units of expression from two biological replicates (R1 and R2) were calculated for each gene by averaging triplicate RT_PCR reactions and normalizing to a control gene, ama-1. Error bars represent the standard error of the mean (SEM) for triplicate RT_PCR reactions. For all nine genes, mes-4 RNAi significantly reduced the relative expression of spr-5; met-2 compared to spr-5; met-2 fed L4440 control RNAi (unpaired t-test, p-value $<0.001$ ).

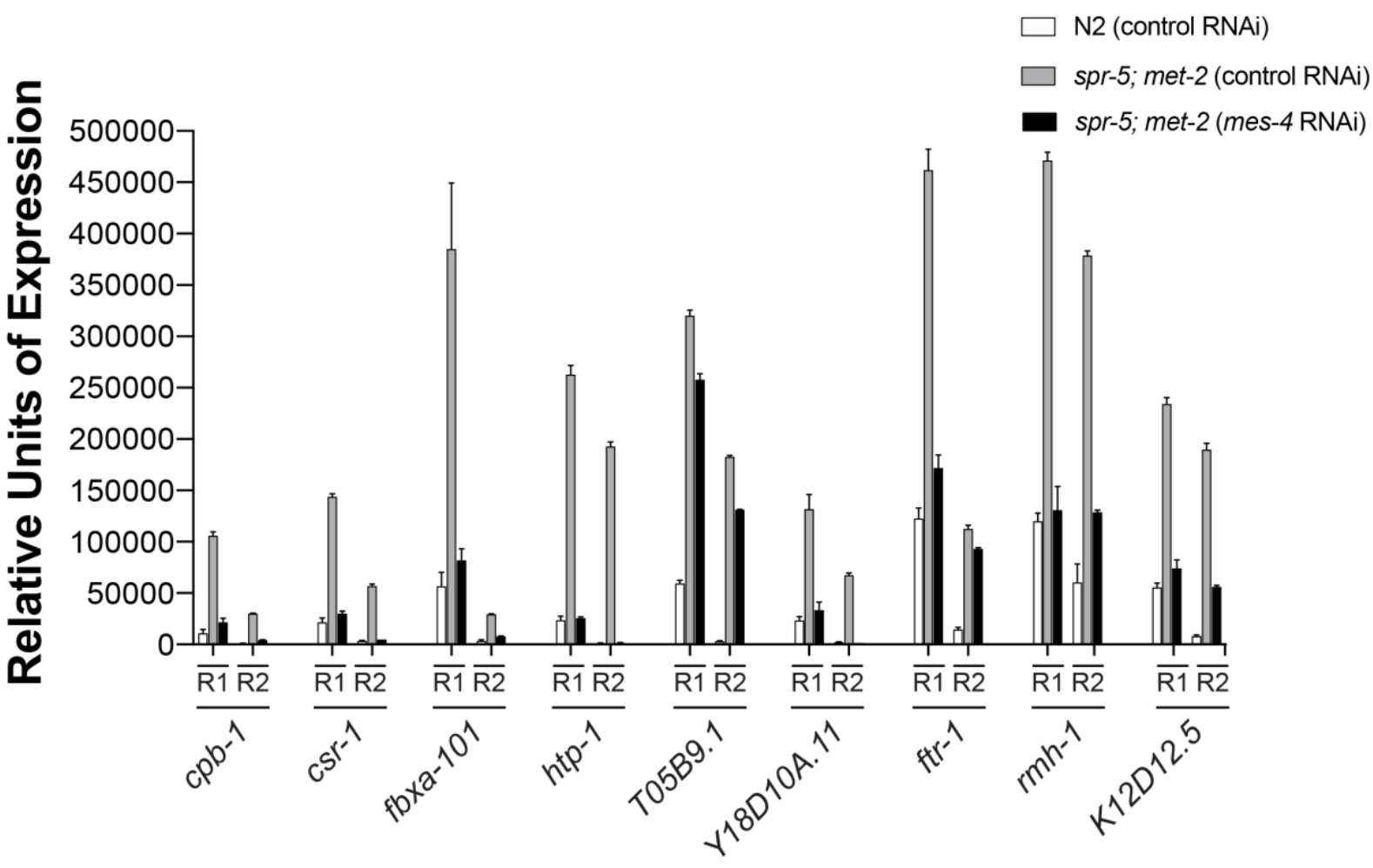

MES-4 Germline Genes 
Figure 6. Knocking down MES-4 rescues developmental delay in spr-5; met-2 progeny. DIC images of N2 (A-D) or spr-5; met-2 (E-H) progeny from hermaphrodite parents treated with control (L4440 vector only) RNAi (A, E), mes-4 RNAi (B, F), met-1RNAi (C, G), or set-2 RNAi (D, H) 72 hours post synchronized lay. Scale bar: $100 \mu \mathrm{m}$. (I) Quantification of the number of progeny (represented as \% Adult Progeny) from A-H that made it to adults by 72 hours. Error bars represent the standard deviation of the mean from two or three experiments. $\mathrm{N}$ represents the total number of progeny from 30-40 hermaphrodites scored across independent experiments. (unpaired t-test, ${ }^{* * * *}$ represent a p-value $<0.0001$ ).
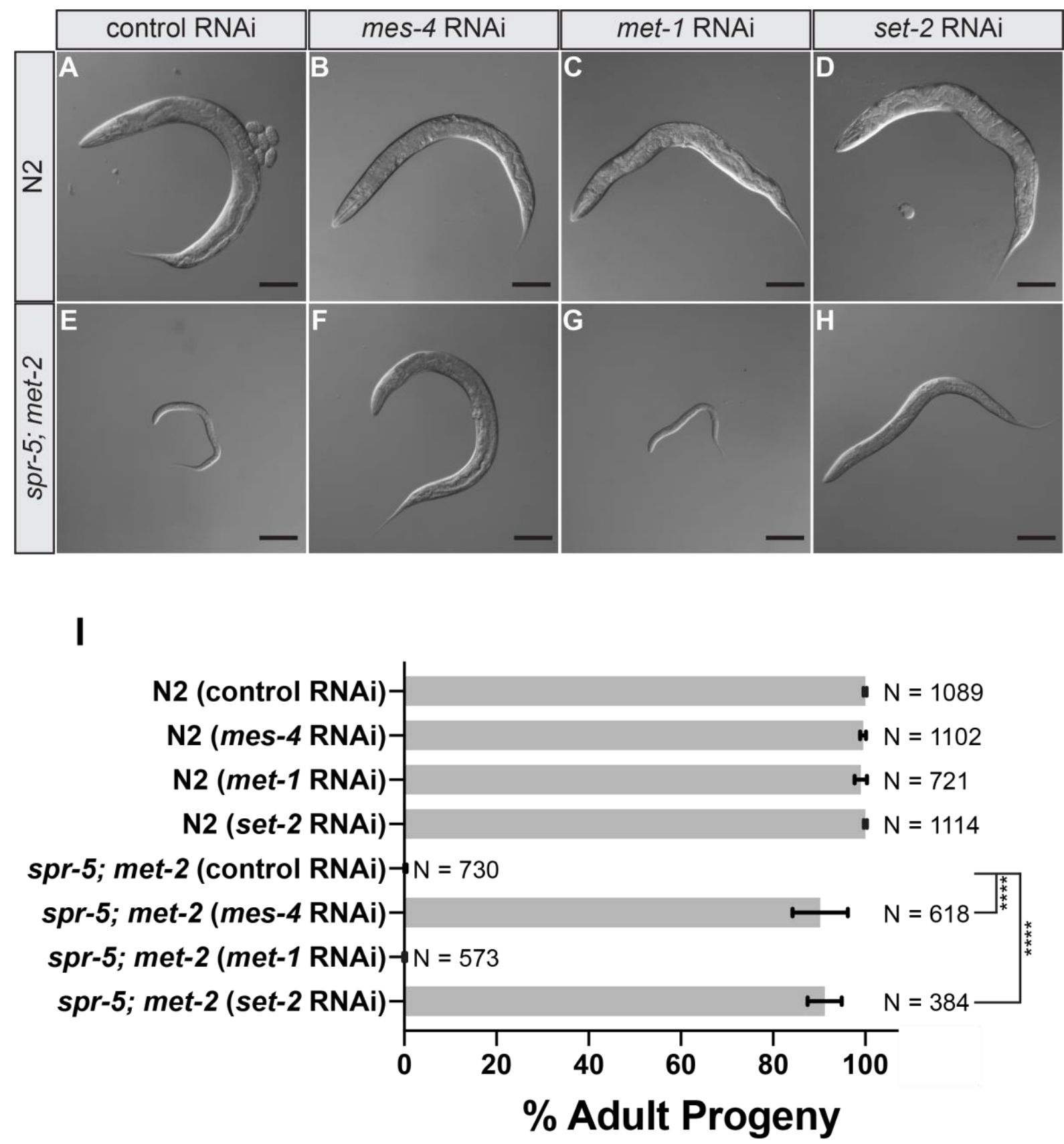
Figure 7. let-858 transgene silencing in the soma of spr-5; met-2 mutants. $40 \mathrm{x}$ differential contrasting interference (DIC) (A, C, E, G, and I) and immunofluorescent (B, D, F, $\mathrm{H}$, and $\mathrm{J}$ ) images of N2 (A-D) and spr-5; met-2 (E-J) L2 progeny. Arrows denote faint expression of LET-858::GFP. Scale bar: $50 \mu \mathrm{m}$. Scale bar is the same for all panels. Percentage of animals where the expression level of LET-858::GFP was scored as either bright expressing (bright green, representative shown in panel B and F), faint expressing (dark green, representative shown in panel $\mathrm{D}$ and $\mathrm{H}$ ), or not expressing (black, representative shown in panel J) in $\mathrm{N} 2(\mathrm{~N}=132)$ versus spr-5; met-2 ( $\mathrm{N}=87)$ progeny $(\mathrm{K})$. The quantification represents the percentages of LET-858::GFP expressing progeny from two independent experiments. To control for the segregation of the let-858 transgene, progeny scored as "off" were normalized for the presence of the let-858 transgene in animals as detected by PCR (see methods).
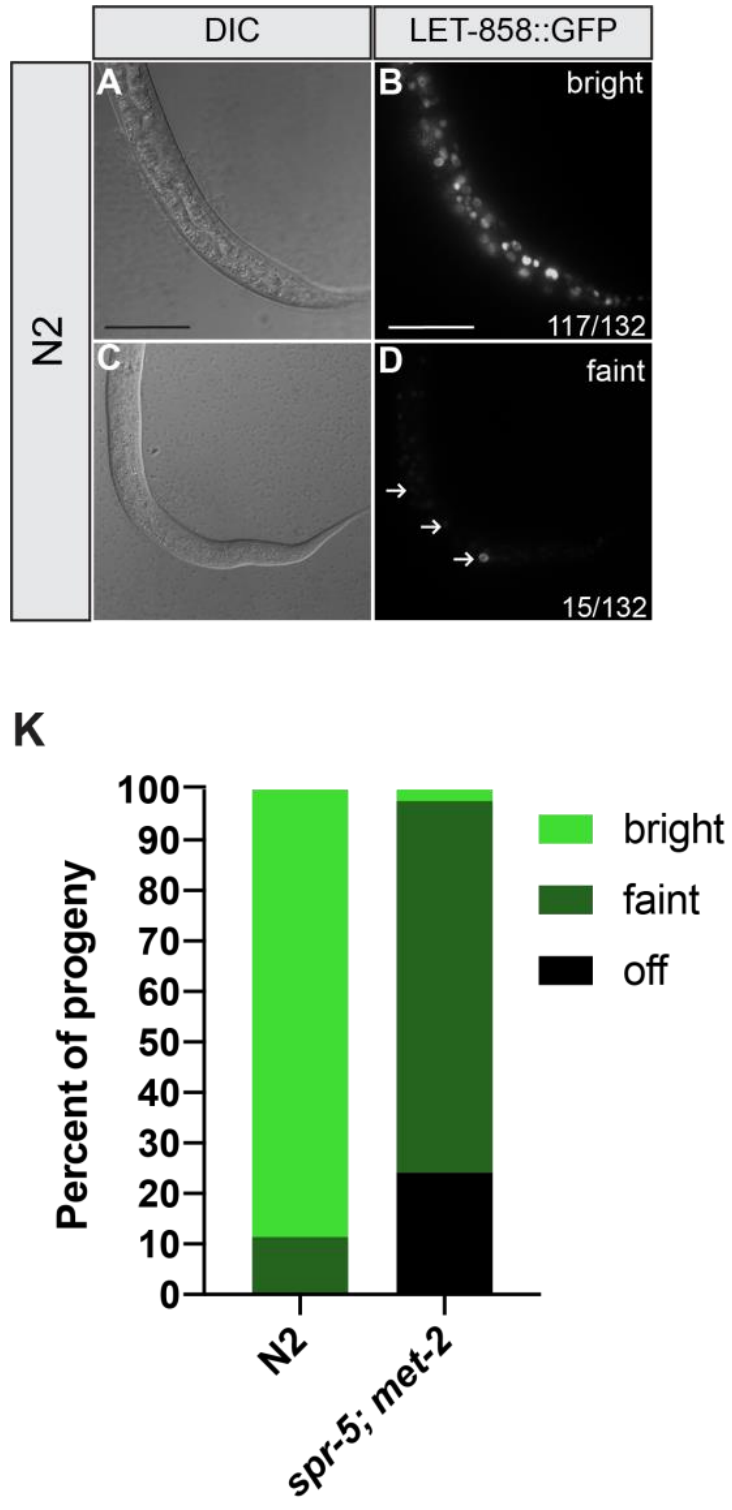

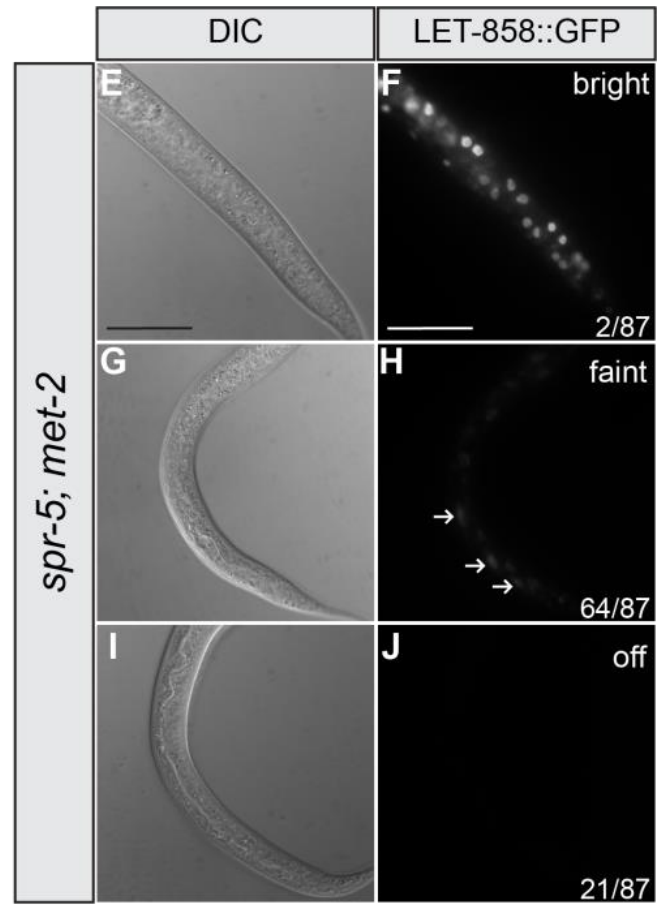


Figure 8: A model for how maternal reprogramming of inherited histone methylation helps to specify germline versus soma. During development, SET- 2 and MET-1 add transcriptionally coupled H3K4me1/2 and H3K36me2/3 to germline expressed genes in the parental germline, respectively. (A) At fertilization, these germline expressed genes undergo maternal epigenetic reprogramming (establishment phase) by SPR-5 and MET- 2 to remove H3K4me1/2 and add H3K9me1/2. In the germline blastomeres of the embryo, PIE-1 prevents global transcription by inhibiting POL-II. In the absence of transcription, MES-4 maintains H3K36me2/3 at MES-4 germline genes that have acquired transcriptionally coupled $\mathrm{H} 3 \mathrm{~K} 36 \mathrm{me} 2 / 3$ in the previous germline. This enables these genes to avoid being repressed by maternal spr-5; met-2 reprogramming and ensures that these genes remain bookmarked for re-expression once the germline begins to proliferate later in development. In addition, multiple systems, such as LIN-15B and LIN-35, as well as MEP1 and LET-418, function in somatic tissues to further antagonize H3K36 bookmarking by MES-4 (maintenance phase). (B) Without SPR-5 and MET-2 maternal reprogramming, $\mathrm{H} 3 \mathrm{~K} 4 \mathrm{me} 1 / 2$ is inappropriately inherited in somatic tissues, allowing MES-4 to ectopically add H3K36me2/3 at these germline genes. This leads to ectopic expression of MES-4 germline genes in somatic tissues and a severe developmental delay. Orange circles represent somatic cells, Grey circles outlined in blue dashed-lines represent germ cells, and orange circles outlined in dashed-blue lines depict somatic cells that ectopically express MES-4 germline genes. P-lineage, germline blastomeres, are indicated by the letter $\mathrm{P}$, and the primordial germline cells are indicated by $\mathrm{Z} 2$ and $\mathrm{Z3}$.

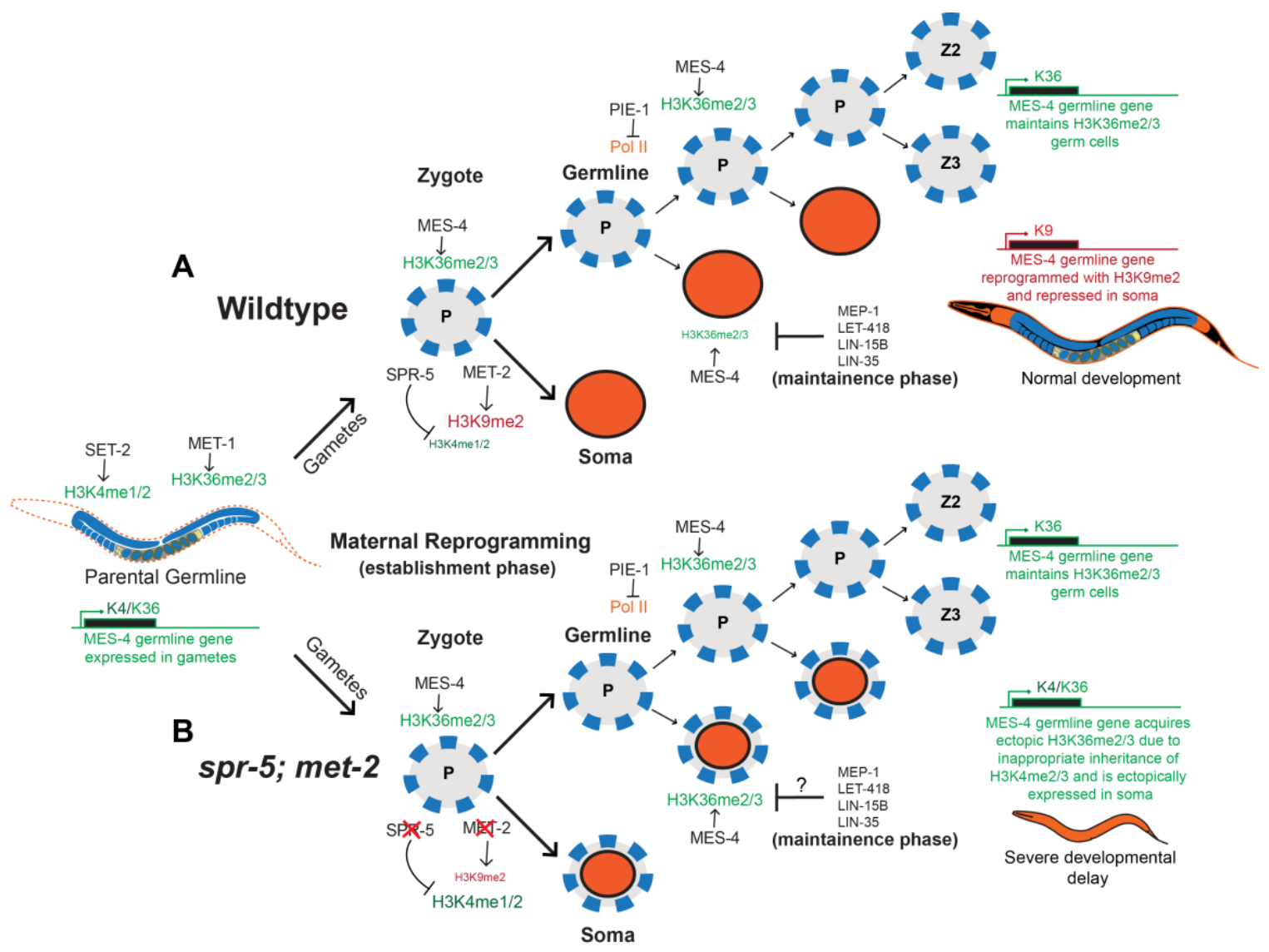


Figure 1 - supplement figure 1. spr-5; met-2 mutants display severe developmental delay and protruding vulva. 40x DIC images of N2 (A), spr-5 (B), met-2 (C), and spr-5; met-2 (D) progeny at 72 hours post synchronized lay. DIC image in (D) was magnified an additional 4x. 40x DIC images of two examples of spr-5; met-2 adult progeny (E, F) at seven days post synchronized lay. Dashed-line (D) outlines germline and asterisks (E, F) denote protruding vulva. Scale bar: $100 \mu \mathrm{m}$. (G) Quantification of spr-5; met-2 progeny that reached the adult stage by seven days post synchronized lay, along with quantification of protruding vulva ( $\mathrm{Pvl}$ ) in these animals that reached the adult stage. The 876 progeny scored came from a total of 25 hermaphrodites across two independent experiments.
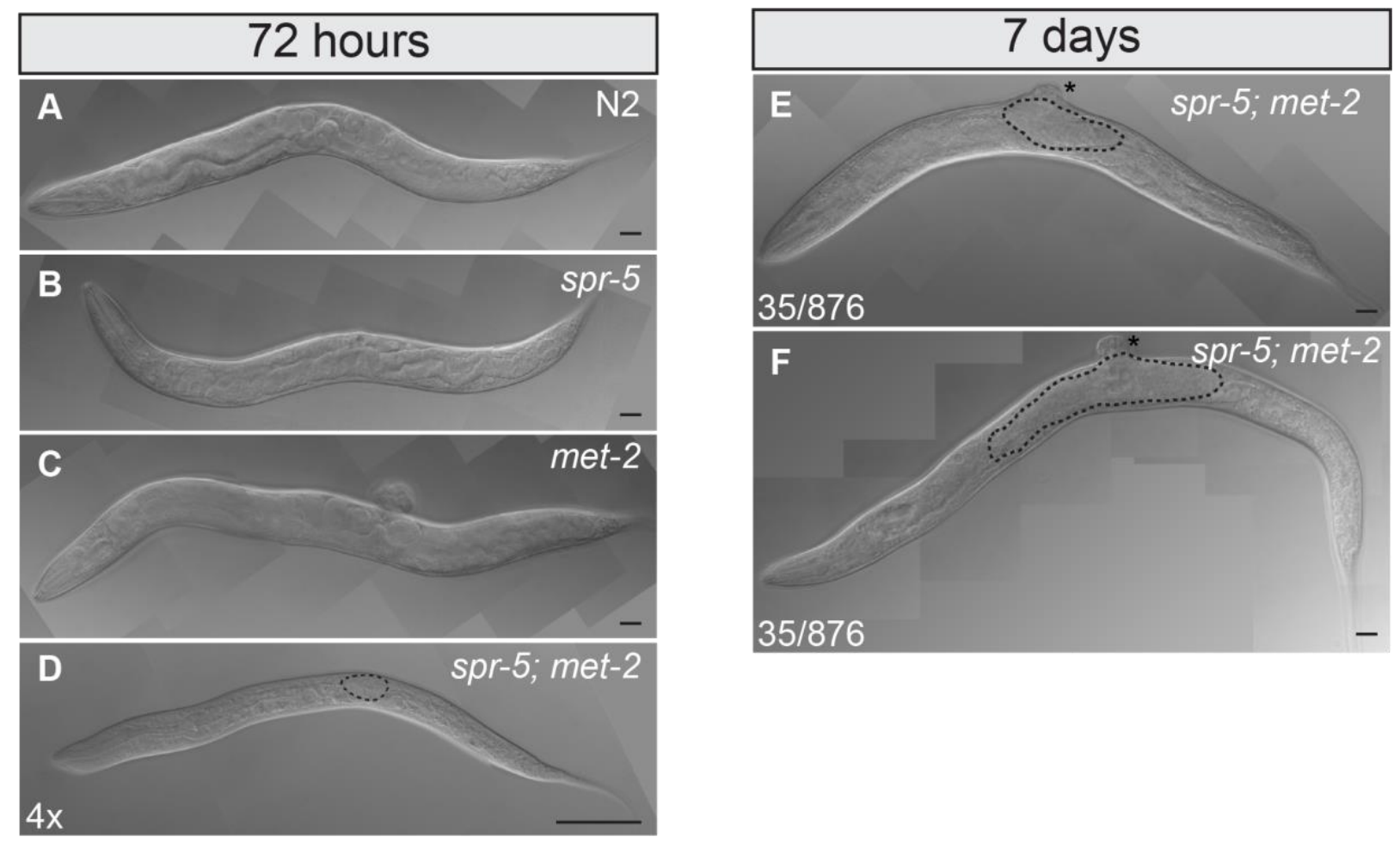

G

\begin{tabular}{|c|c|c|c|c|}
\hline $\begin{array}{c}\text { Total \# of } \\
\text { progeny scored }\end{array}$ & $\begin{array}{c}\text { Total \# of } \\
\text { adult progeny }\end{array}$ & $\begin{array}{c}\text { Percent of } \\
\text { adult progeny }\end{array}$ & $\begin{array}{c}\text { Total \# of } \\
\text { adult progeny } \\
\text { with Pvl }\end{array}$ & $\begin{array}{c}\text { Percent of } \\
\text { adult progeny } \\
\text { with Pvl }\end{array}$ \\
\hline 876 & 35 & $3.9 \%$ & 31 & $88.5 \%$ \\
\hline
\end{tabular}


Figure 2 - supplement figure 1. Differential gene expression in spr-5, met-2, and spr5; met-2 progeny compared to $\mathbf{N} 2$ progeny. (A) Table summary of differentially expressed genes in spr-5, met-2, and spr-5; met-2 L1 progeny from DESEQ2 analysis (significance cut-off of p-value $<0.05$ ). (B) Overlap of differentially expressed genes between spr-5, met-2, and spr-5; met-2 L1 progeny. Gene Ontology analysis showing Biological Processes (C) and Cellular Components (D) amongst genes that were upregulated (red) and down-regulated (green) in spr-5; met-2 L1 progeny compared to N2. Combined Score was computed to determine gene set enrichment (Chen et al., 2013) (see source data file 1 for gene ontology R scripts).

A

\begin{tabular}{|c|c|c|c|}
\hline Genotype & $\begin{array}{c}\text { Total \# of } \\
\text { differentially } \\
\text { expressed genes }\end{array}$ & Up-regulated & Down-regulated \\
\hline spr-5 & 343 & 259 & 84 \\
\hline met-2 & 413 & 262 & 151 \\
\hline spr-5; met-2 & 778 & 482 & 296 \\
\hline
\end{tabular}

C

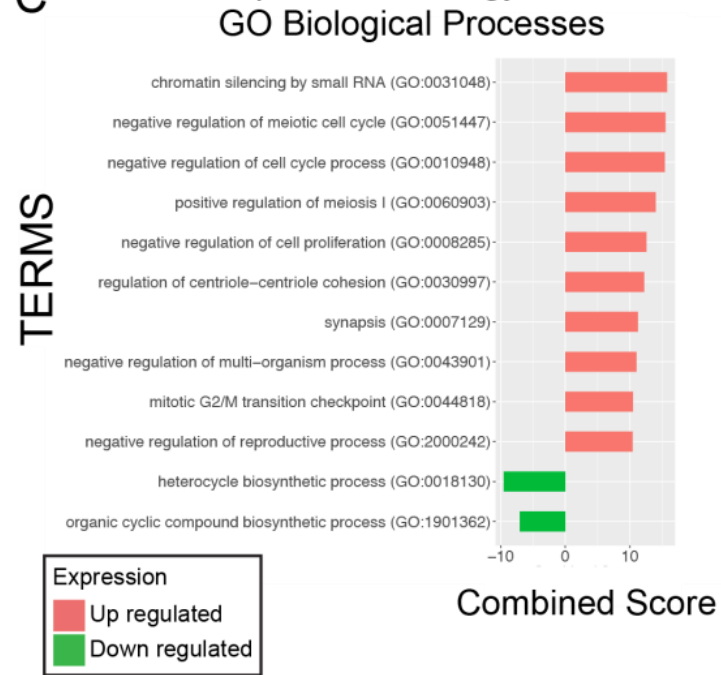

B

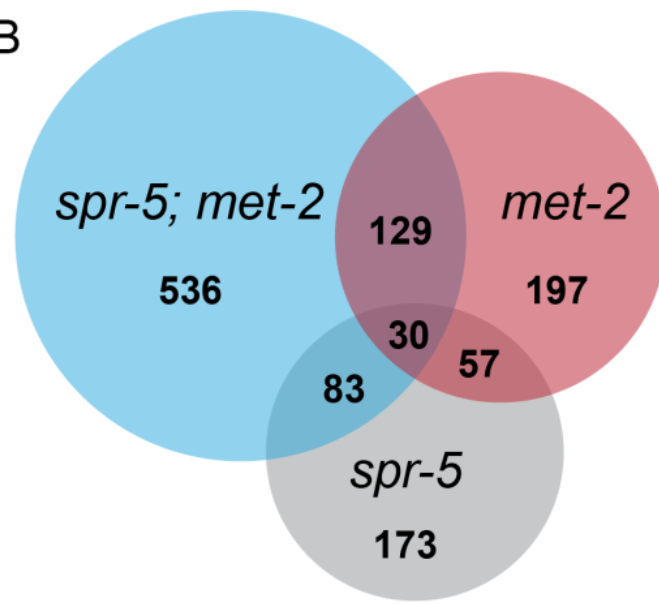

D Top Gene Ontology
GO Cellular Components

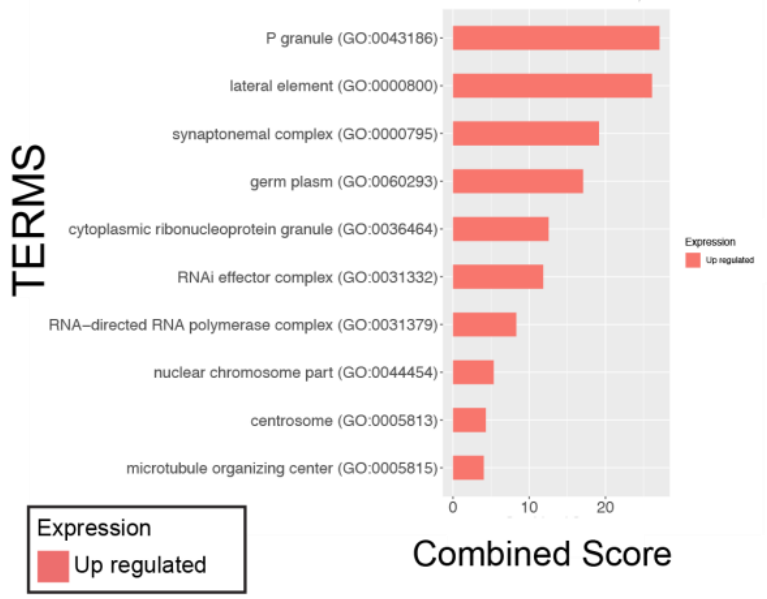


Figure 2 - supplement figure 2. Differential expression and replicate comparison of RNAseq experiments performed on N2, spr-5; met-2, and spr-5; met-2 L1 progeny. Volcano plot of log2 fold changes in gene expression ( $\mathrm{x}$-axis) by statistical significance (-Log10 P-value; y-axis) in spr-5 (A), met-2 (B), and spr-5; met-2 (C) L1 Progeny compared to N2 (see source data file 2 for volcano plot R scripts). Heatmap of differentially expressed RNA-seq transcripts between N2 and spr-5 (D), met-2 (E), and spr-5; met-2 (F). Data was scaled and hierarchical clustering was performed using the complete linkage algorithm. Distance was measured by calculating pairwise distance. Higher (red) and lower (green) expression is reported as a z-score. (see source data file 3 for heatmap R scripts).

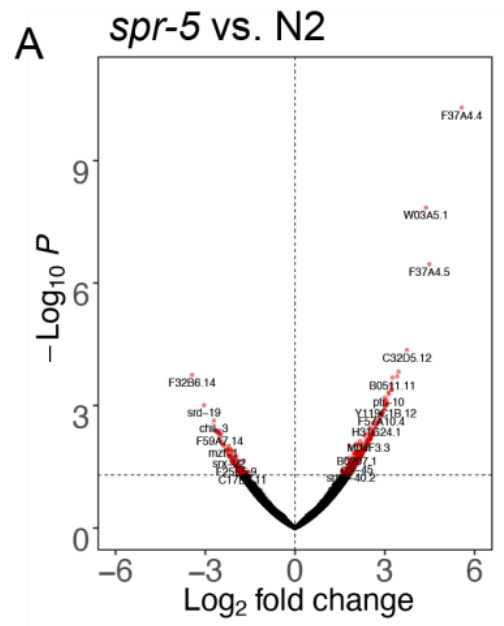

D

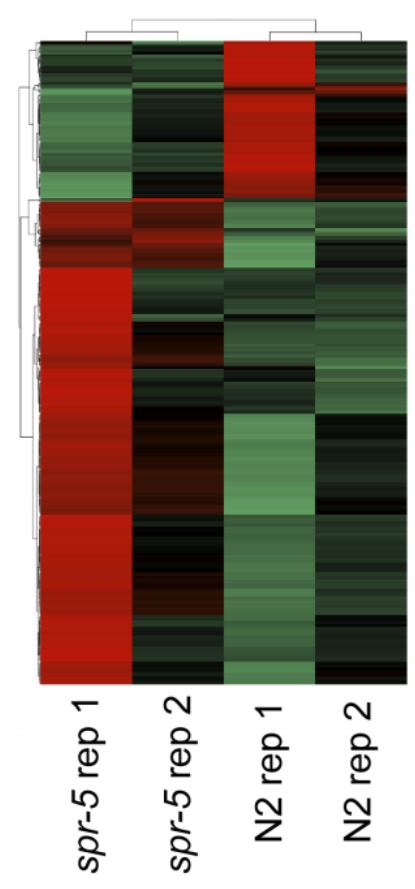

$\mathrm{B}$

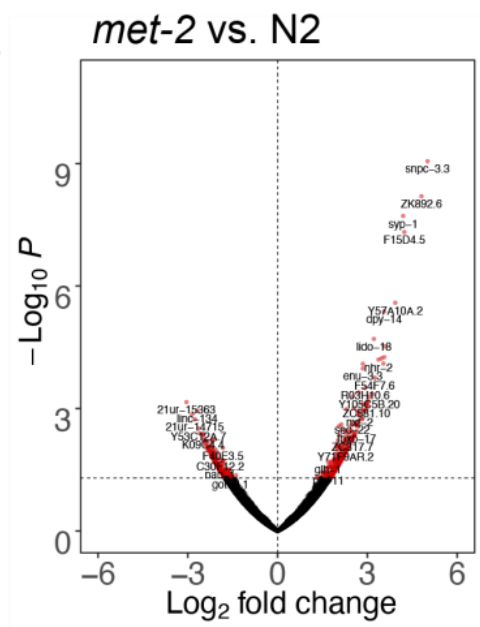

$E$

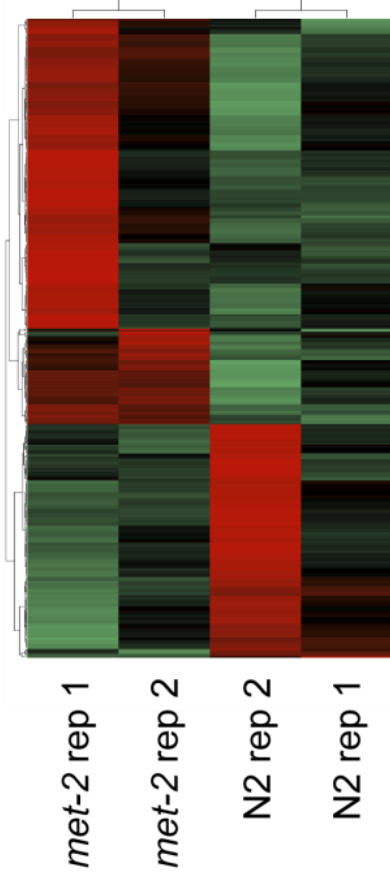

C

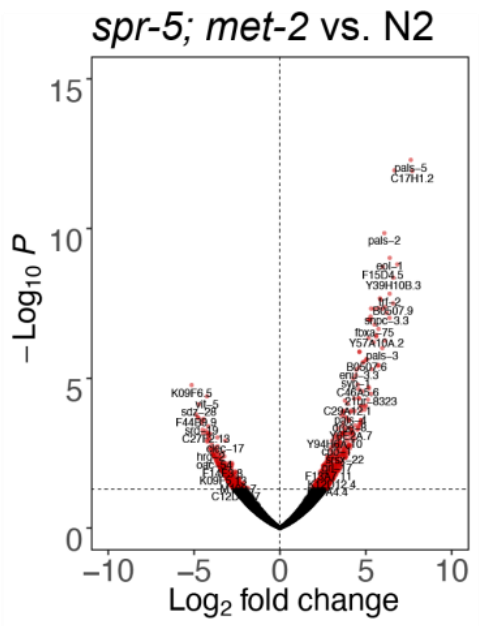

$\mathrm{F}$

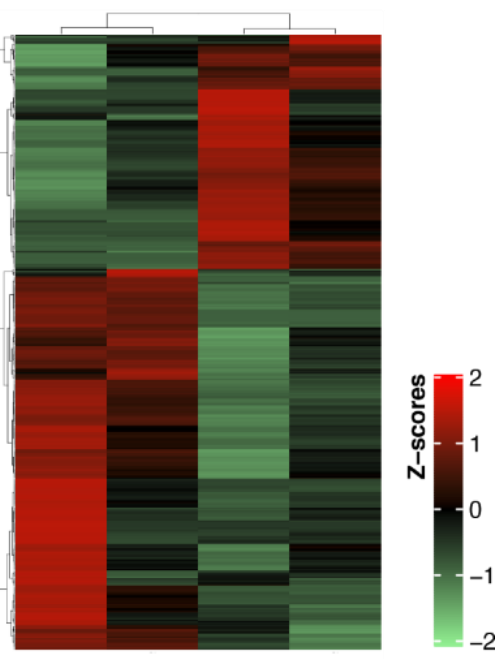

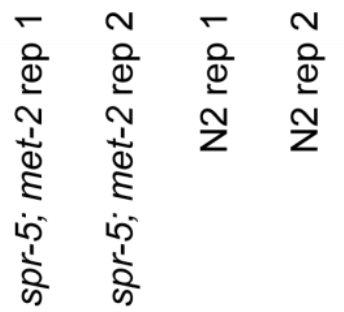


Figure 2-supplemental figure 3. MES-4 germline genes are ectopically expressed in spr-5; met-2 mutant soma in the RNAseq repeat experiment. Overlap between MES-4 germline genes and genes up-regulated (A) and down-regulated (B) in spr-5; met-2 L1 progeny. Significant over-enrichment in A was determined by the hypergeometric test ( ${ }^{*} \mathrm{P}$ value < 1.20E-54). (C) Heatmap of log2 fold change (FC) of 176 MES-4 germline genes in spr-5; met-2 mutants compared to $\mathrm{N} 2$ (2 replicates, low-input, see methods) vs. repeat of spr-5; met-2* mutants compared to N2 (*3 replicates, standard Poly-A selection, see methods). Log2FC values are represented in a yellow to blue gradient and range from -2 to 5 and were sorted by the average $\log 2 \mathrm{FC}$ in spr-5; met-2 and spr-5; met-2* progeny. Yellow represents genes with negative log2FC values and blue represents genes with positive $\log 2 \mathrm{FC}$ values compared to N2. The remaining $21 \mathrm{MES}-4$ germline genes were not included because they do not have an expression value in one or more of the data sets (spr-5; met-2 or spr-5; met-2*).
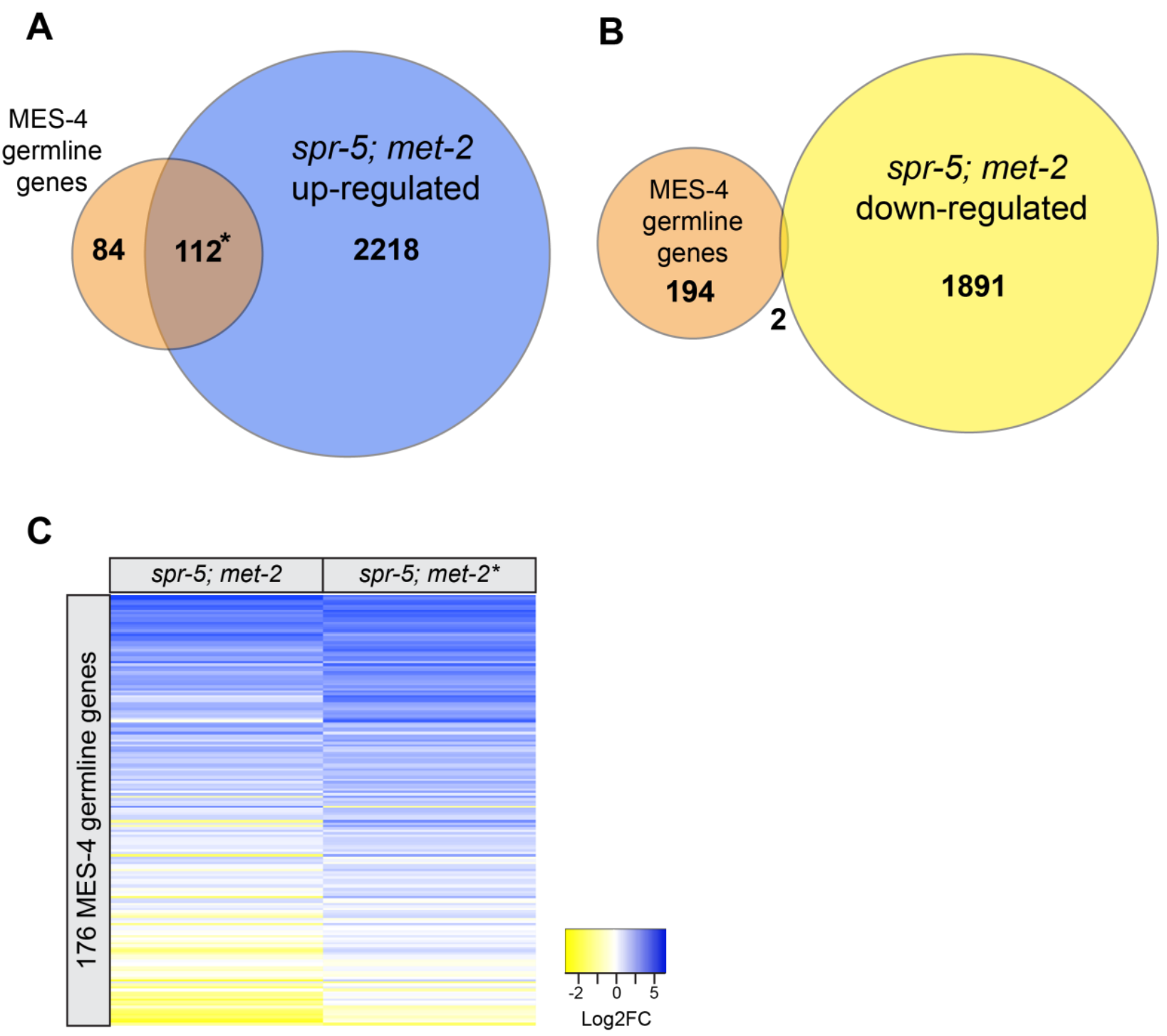
bioRxiv preprint doi: https://doi.org/10.1101/2020.01.22.914580; this version posted May 29, 2020. The copyright holder for this preprint (which

was not certified by peer review) is the author/funder, who has granted bioRxiv a license to display the preprint in perpetuity. It is made available under aCC-BY-NC-ND 4.0 International license.

Figure 3 - supplement figure 1. smFISH control experiments. smFISH images of ama-1 mRNA (A, C, D, F) and a no probe control (G, I, J, L) in N2 (A-C, G-I) and spr-5; met-2 (D-F, JL) L1 progeny. DAPI was used as a nuclear marker (B, C, E, F, H, I, K, L). Scale bar $40 \mu \mathrm{m}$.
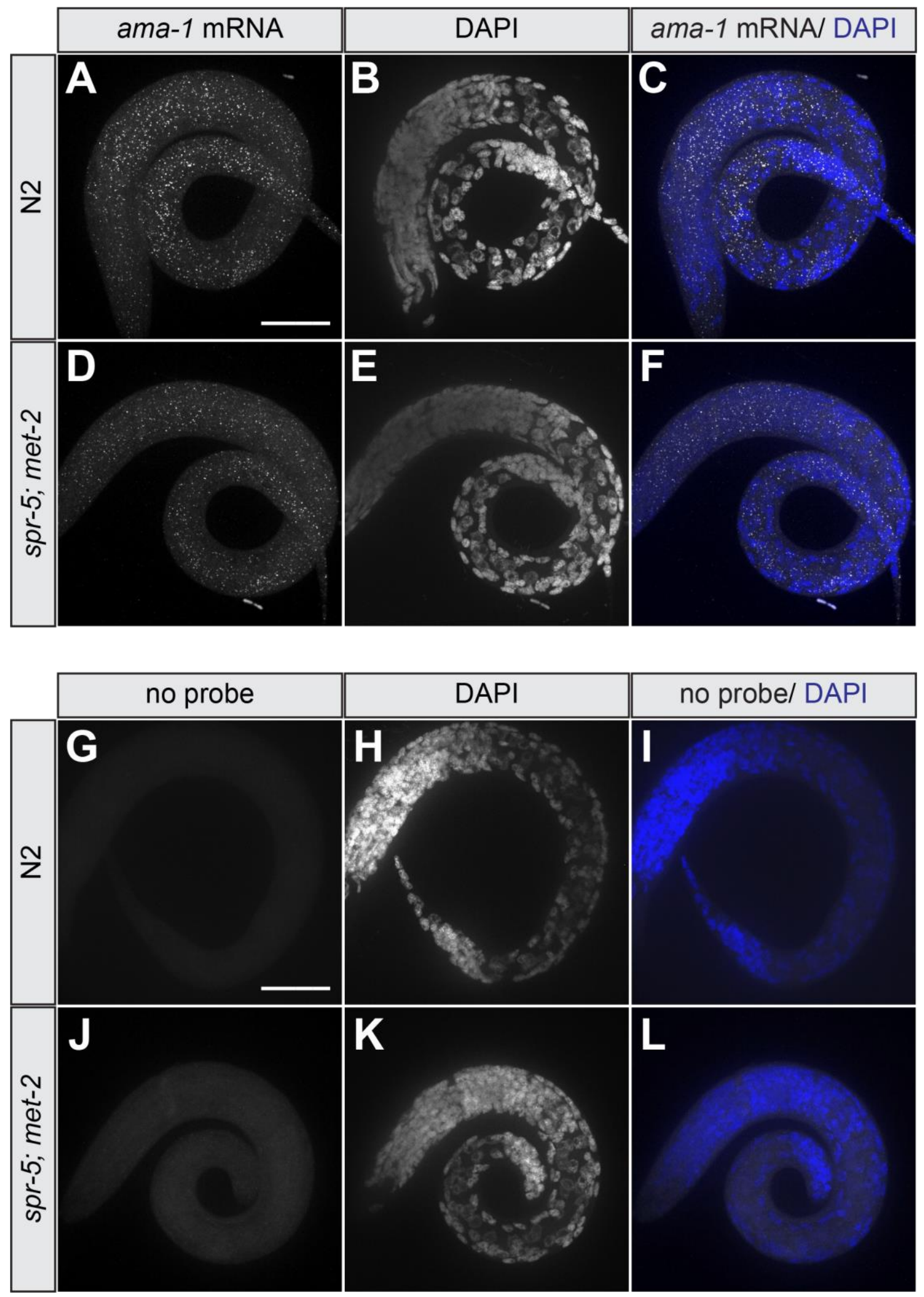
Figure 4 - supplement figure 1. Replicate comparison of H3K36me3 ChIP-seq analysis in spr-5; met-2 L1 progeny. Heatmap of H3K36me3 ChIP-seq reads from a second replicate ( 1 st replicate in Figure 4) normalized to reads per kilobase million (RPKM) over the gene bodies of 196 MES-4 germline genes in wild-type (N2) (A) versus spr-5; met2 (B) L1 progeny. Gene bodies were pseudoscaled to $1 \mathrm{~kb}$ with $500 \mathrm{bp}$ borders separated by orange bars that represent the transcriptional start site (TSS) and transcriptional end site (TES). Integrative Genome Viewer (IGV) image of H3K36me3 ChIP-seq reads from replicate 2 normalized to RPKM at MES-4 germline genes (C-F, J-M) versus control genes (G-I, N-P) in N2 (C-I) and spr-5; met-2 (J-P) L1 progeny. RPKM IGV windows were scaled between 0 and 107 RPKM for all genes. Venn-diagram displaying the overlap of H3K36me3 ChIP-seq called broad peaks for $\mathrm{N} 2(\mathrm{Q})$ and spr-5; met-2 (R) replicates.
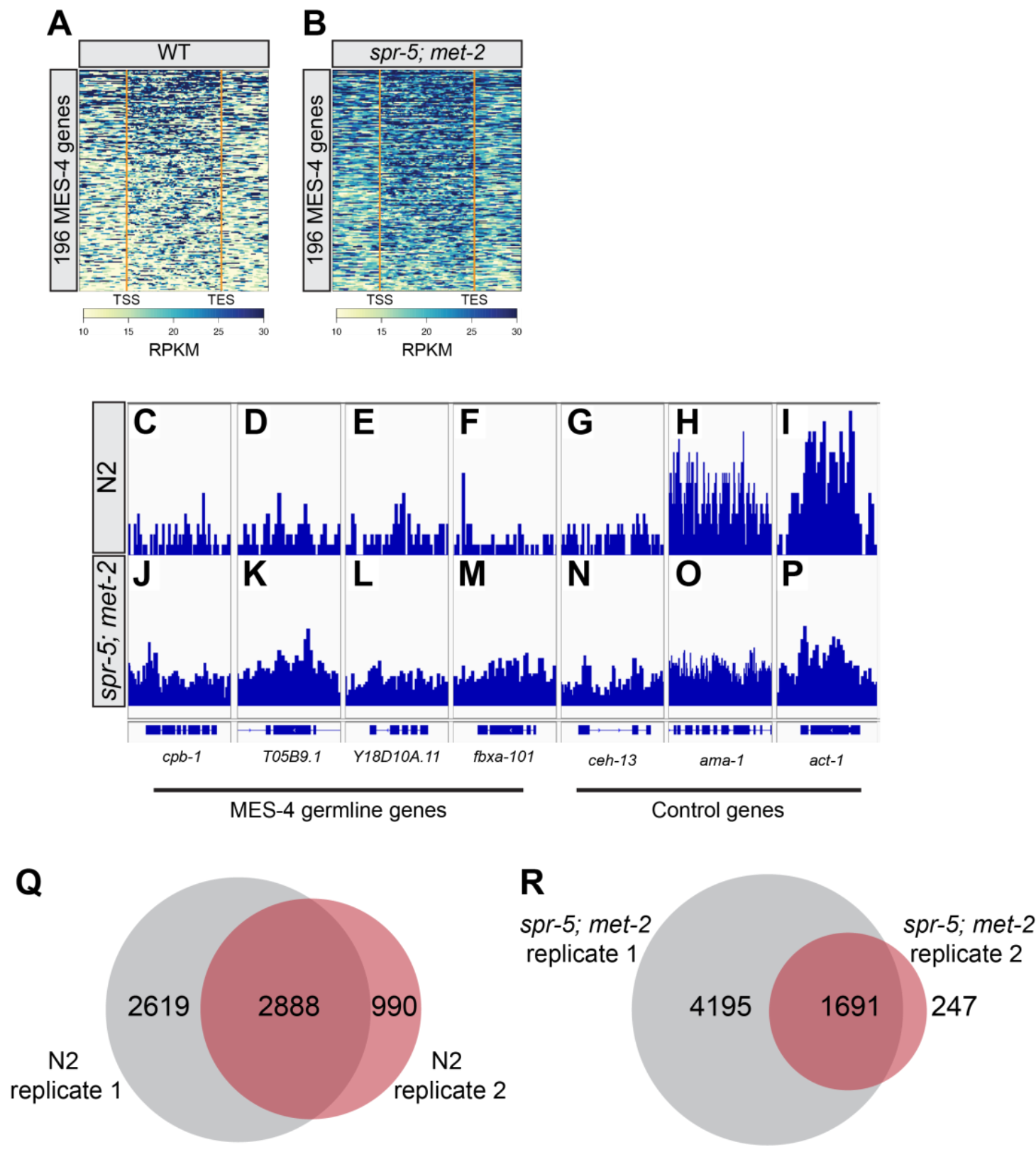
Figure 4 - supplement figure 2. MES-4 germline genes display H3K9me2 at their promoters. Heatmap of H3K9me2 promoter peaks in N2 L1 progeny at all $196 \mathrm{MES}-4$ germline genes (A), and at the $34 \mathrm{MES}-4$ germline genes that were ectopically expressed in the soma of spr-5; met-2 progeny (B). ChIP-seq reads were scaled to genome wide coverage for H3K9me2 (15 million reads). The transcriptional start site (TSS) is denoted by an orange bar with $500 \mathrm{bp}$ flanking regions upstream and downstream of the TSS. Integrative Genome Viewer (IGV) image of ChIP-seq reads from N2 L1 progeny scaled to genome wide coverage for H3K9me2 (15 million reads) over the promoters of MES-4 germline genes that were ectopically expressed in the soma of spr-5; met-2 progeny (C-F) and control genes (G-I). IGV windows were scaled between 0 and 255 RPKM. Asterisks (*) denotes $\mathrm{H} 3 \mathrm{~K} 9 \mathrm{me} 2$ promoter peaks at MES-4 germline genes.
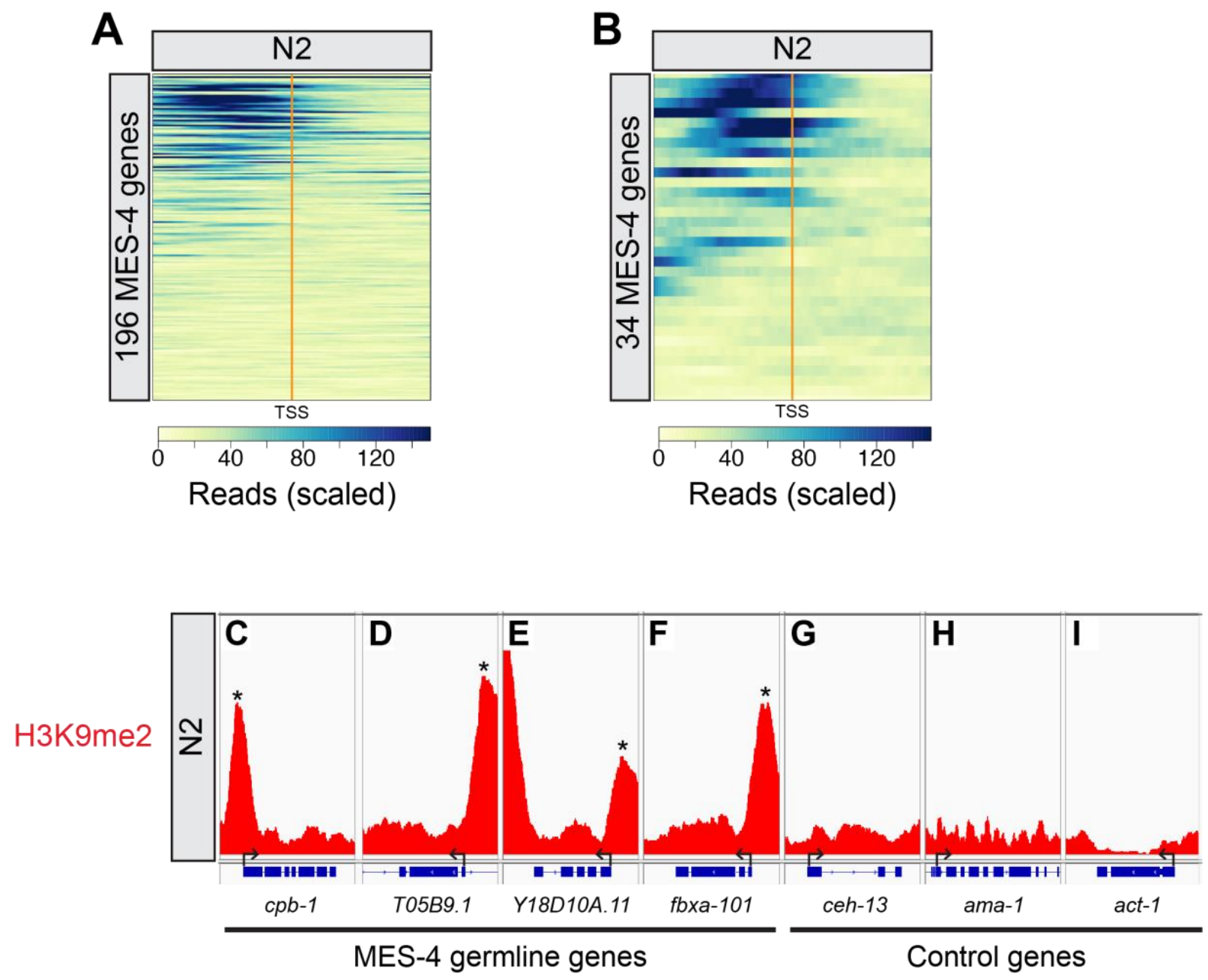
Supplementary file 1. Raw scores for developmental delay in N2, spr-5, met-2, and spr-5; met-2 progeny. Progeny were scored 72 hours after a synchronized lay as developing to the L1, L2, L3, L4, or young adult (YA) stage (Figure 1A-E). N= the total number of progeny from a total of 20-25 hermaphrodites scored over three experiments.

Supplementary file 2. Differentially expressed transcripts in spr-5, met-2, and spr-5; met-2 progeny. List of differentially expressed transcripts in spr-5, met-2, spr-5; met-2 (Wald test, P-value<0.05), and the repeat RNA-seq experiment with three additional spr-5; met-2 replicates (Wald test, $\mathrm{P}$-adj $<0.05$ ) $\mathrm{L} 1$ progeny compared to $\mathrm{N} 2$ progeny from DESEQ2 analysis (Figure 2-supplement figure 2A-D).

Supplementary file 3. 176 MES-4 germline gene $\log 2$ (FC) values used to generate heatmap. The $\log 2(\mathrm{FC})$ values of the $176 \mathrm{MES}-4$ germline genes from the DESEQ2 analysis performed on spr-5, met-2, spr-5; met-2, and the repeat RNA-seq experiment of spr-5; met-2 $(*)$ L1 progeny compared to N2 progeny. $\log 2(\mathrm{FC})$ values were sorted from highest to lowest based on the differential expression of genes in spr-5; met-2 progeny compared to N2 (Figure 2C-D).

Supplementary file 4. smFISH probe sequences. List of smFISH probe sequences for $h t p$ 1 and $c p b-1$ used in smFISH experiments (Figure 3).

Supplementary file 5. Primer sequences used for genotyping and RT-PCR. List of genotyping primers and RT-PCR primers (Figure 5).

Supplementary file 6. Raw values from Quantitative RT-PCR analysis. Raw SQ means and standard deviations for RT-PCR experiments (Figure 5).

Source data file 1. R scripts for gene ontology (Figure 2-supplement figure 1C-D)

Source data file 2. R scripts for volcano plots (Figure 2-supplement figure 2A-C)

Source data file 3. R scripts for heatmaps (Figure 2-supplement figure 2D-F) 